\title{
On Lorentz-invariant bispin-2 theories
}

\author{
Rampei Kimura, ${ }^{1}$ Atsushi Naruko $\odot,{ }^{2}$ and Daisuke Yamauchi ${ }^{3}$ \\ ${ }^{1}$ Waseda Institute for Advanced Study, Waseda University, \\ 1-6-1 Nishi-Waseda, Shinjuku, Tokyo 169-8050, Japan \\ ${ }^{2}$ Center for Gravitational Physics, Yukawa Institute for Theoretical Physics, Kyoto University, \\ Kyoto 606-8502, Japan \\ ${ }^{3}$ Faculty of Engineering, Kanagawa University, \\ Kanagawa-ku, Yokohama-shi, Kanagawa 221-8686, Japan
}

(Received 7 January 2021; accepted 12 July 2021; published 9 August 2021)

\begin{abstract}
We investigate a Lorentz invariant action that is quadratic in two rank-2 symmetric tensor fields in Minkowski spacetime. We apply a scalar-vector-tensor decomposition to two tensor fields by virtue of three-dimensional rotation invariance of Minkowski spacetime and classify theories with 7 degrees of freedom based on the Hamiltonian analysis. We find two new theories, which cannot be mapped from the linearized Hassan-Rosen bigravity. In these theories, the new mass interactions can be allowed thanks to the transverse diffeomorphism invariance of action.
\end{abstract}

DOI: 10.1103/PhysRevD.104.044021

\section{INTRODUCTION}

The attempt to seek ghost-free massive gravity theories has again attracted considerable attention by the discovery of de Rham-Gabadadze-Tolley (dRGT) massive gravity [1]. The first attempt of constructing massive spin-2 theory has been carried out by Fierz and Pauli, and it is the quadratic action for a massive spin-2 particle in a flat spacetime [2]. Once we embed this into a curved spacetime, the behavior of the massive spin-2 field does not smoothly connect to the well-known massless one, i.e., the linearized general relativity $[3,4]$. The discontinuity found by van Dam, Veltman, and Zhakarov turned out to be an artifact of the truncation at linear order, and the massive spin-2 theory, in fact, has the continuous massless limit when taking into account nonlinearities as pointed out by Vainshtein [5]. Nonetheless, an unwanted degree of freedom (DOF), Boulware-Deser ghost [6], which is absent at linear order, reappears at the nonlinear level, and it unfortunately behaves as Ostrogradsky's ghost [7]. In dRGT massive gravity, such an unwanted degree of freedom is successfully eliminated by the careful choice of nonlinear potential terms $[1,8]$. Although the dRGT massive gravity possesses the cosmological constant solution in a cosmological background [9], it is perturbatively unstable [10,11]. For this reason, one needs to seek a ghost-free extension of

Published by the American Physical Society under the terms of the Creative Commons Attribution 4.0 International license. Further distribution of this work must maintain attribution to the author(s) and the published article's title, journal citation, and DOI. Funded by SCOAP ${ }^{3}$. massive gravity which should be at least cosmologically viable and stable. Such an attempt without introducing an extra DOF has been investigated, taking into account derivative interactions [12-14] and metric transformation [15], but unfortunately most of them are not successful. Recently, by breaking the translation invariance of the Stückelberg field, new extended theories of massive gravity have been found, and their cosmological perturbations are stable around cosmological backgrounds [16-18].

Another way to extend massive gravity is to introduce the second dynamical symmetric tensor field. In massive gravity theories, to give mass to graviton, in addition to the metric $g_{\mu \nu}$, one needs to introduce the so-called reference metric $f_{\mu \nu}$, which is usually taken to be a Minkowski metric. In massive bigravity theories, the reference metric can be promoted to be a dynamical tensor field by introducing its kinetic terms. The simplest extension of dRGT massive gravity is proposed by Hassan and Rosen by adding the Einstein-Hilbert kinetic terms even for the second metric [19]. In Hassan-Rosen bigravity, the total number of physical DOFs is 7 , which consists of 2 from a massless graviton and 5 from a massive graviton. This fact can easily be seen by expanding both metrics around Minkowski spacetime, that is, $g_{\mu \nu} \rightarrow \eta_{\mu \nu}+$ $h_{\mu \nu} / M_{g}$ and $f_{\mu \nu} \rightarrow \eta_{\mu \nu}+f_{\mu \nu} / M_{f}$, where $M_{g}$ and $M_{f}$ are, respectively, the Planck mass for the metric $g_{\mu \nu}$ and $f_{\mu \nu}$. Then the quadratic Lagrangian is given by [19]

$$
\begin{aligned}
\mathcal{L}_{\mathrm{HR}}^{(2)}= & -\left(h_{\mu \nu} \hat{\mathcal{E}}^{\mu \nu \alpha \beta} h_{\alpha \beta}+f_{\mu \nu} \hat{\mathcal{E}}^{\mu \nu \alpha \beta} f_{\alpha \beta}\right) \\
& -\frac{m^{2} M_{\mathrm{eff}}^{2}}{4}\left[\left(\frac{h^{\mu}{ }_{\nu}}{M_{g}}-\frac{f^{\mu}{ }_{\nu}}{M_{f}}\right)^{2}-\left(\frac{h}{M_{g}}-\frac{f}{M_{f}}\right)^{2}\right] .
\end{aligned}
$$


Here $\hat{\mathcal{E}}^{\mu \nu \alpha \beta}$ is the linearized Einstein-Hilbert kinetic operator defined as

$$
\begin{aligned}
\hat{\mathcal{E}}_{\alpha \beta}^{\mu \nu}= & {\left[\eta_{\alpha}^{(\mu} \eta_{\beta}^{\nu)}-\eta^{\mu \nu} \eta_{\alpha \beta}\right] \square-2 \partial^{(\mu} \partial_{(\alpha} \eta_{\beta)}^{\nu)} } \\
& +\partial^{\mu} \partial^{\nu} \eta_{\alpha \beta}+\partial_{\alpha} \partial_{\beta} \eta^{\mu \nu}
\end{aligned}
$$

where the round brackets denote the symmetrization of indices, $m$ is the mass of graviton, and the effective Planck mass is given by $M_{\text {eff }}^{2}=\left(1 / M_{g}^{2}+1 / M_{f}^{2}\right)^{-1}$. The mixing terms between $h$ and $f$ in the mass terms can be removed by introducing the linear combination of two metrics,

$$
\begin{aligned}
\frac{1}{M_{\mathrm{eff}}} u_{\mu \nu} & \equiv \frac{1}{M_{f}} h_{\mu \nu}+\frac{1}{M_{g}} f_{\mu \nu}, \\
\frac{1}{M_{\mathrm{eff}}} v_{\mu \nu} & \equiv \frac{1}{M_{f}} h_{\mu \nu}-\frac{1}{M_{g}} f_{\mu \nu} .
\end{aligned}
$$

Then the Lagrangian becomes

$$
\begin{aligned}
\mathcal{L}= & -\left(u_{\mu \nu} \hat{\mathcal{E}}^{\mu \nu \alpha \beta} u_{\alpha \beta}+v_{\mu \nu} \hat{\mathcal{E}}^{\mu \nu \alpha \beta} v_{\alpha \beta}\right) \\
& -\frac{m^{2}}{4}\left(v^{\mu \nu} v_{\mu \nu}-v^{2}\right) .
\end{aligned}
$$

This clearly shows that Hassan-Rosen bigravity at linear order consists of the linearized general relativity for $u_{\mu \nu}$ and the Fierz-Pauli theory for $v_{\mu \nu}$. The absence of the BoulwareDeser ghost has been proved in [20].

Since the construction of bigravity theory is inspired by the dRGT theory, it is not trivial whether the mass interaction of Hassan-Rosen bigravity is unique or not. As for the uniqueness of the dRGT mass term in linear massive gravity theories, see also [21]. For this reason, one might be able to find a new type of mass interactions in bimetric gravity theories. However, such a construction would be extremely difficult to start with a curved spacetime. To this end, in this paper, we investigate a theory with a bispin-2 particle in a flat spacetime, which could represent the linear expansion of a certain nonlinear massive bigravity.

This paper is organized as follows. In Sec. II, we give an action for two rank-2 tensor fields in our setup and decompose them into scalar, vector, and tensor sectors based on transformation properties of tensors with respect to a three-dimensional spatial rotation. In Sec. III, we give ghost-free conditions for the tensor mode. In Sec. IV, we perform the Hamiltonian analysis and derive the conditions to have 2 physical DOFs in the vector sector. In Sec. V, we investigate the scalar sector and classify theories with 1 scalar DOFs. Section VI is devoted to the summary. In Appendix A, we introduce a linear field redefinition and investigate the reduction of the model parameter space. In Appendix B, we provide an explicit expression of the Lagrangian in the scalar sector. In Appendix C, we perform the Hamiltonian analysis of the vector sector with two primary constraints. In Appendix D, we investigate the scalar sector with two primary constraints.

\section{SETUP}

In the present paper, we consider a Lorentz invariant action for two rank-2 symmetric tensor fields, $h_{\mu \nu}$ and $f_{\mu \nu}$, and consider the most general quadratic action which contains up to two derivatives with respect to spacetime for each term. ${ }^{1}$ In general, these symmetric tensor fields possess 20 DOFs in total, and therefore we should impose some conditions to eliminate unwanted DOFs, which could behave as a ghost. Because of the complexity of the analysis, we only focus on theories with 7 physical DOFs, namely $2 \times 2$ (tensor) +2 (vector) +1 (scalar) DOFs, as in the Hassan-Rosen bigravity [19] that consists of massless and massive spin-2 fields in the linearized limit. As preparation for later analysis, in this section, we introduce the generic action for the bispin-2 tensor field and scalar-vector-tensor decomposition of it. For the Hamiltonian analysis in Fourier space, we follow the procedure developed in [22-24] and adopt the notation in [21].

\section{A. Double spin-2 theory}

Let us consider a generic action for two rank-2 symmetric tensor fields up to the quadratic order in fields around Minkowski spacetime,

$$
\begin{aligned}
S= & \int \mathrm{d}^{4} x\left(-\mathcal{K}_{h}^{\alpha \beta \mid \mu \nu \rho \sigma} h_{\mu \nu, \alpha} h_{\rho \sigma, \beta}-\mathcal{K}_{f}^{\alpha \beta \mid \mu \nu \rho \sigma} f_{\mu \nu, \alpha} f_{\rho \sigma, \beta}\right. \\
& -\mathcal{G}^{\alpha \beta \mid \mu \nu \rho \sigma} h_{\mu \nu, \alpha} f_{\rho \sigma, \beta}-\mathcal{M}_{h}^{\mu \nu \rho \sigma} h_{\mu \nu} h_{\rho \sigma} \\
& \left.-\mathcal{M}_{f}^{\mu \nu \rho \sigma} f_{\mu \nu} f_{\rho \sigma}-\mathcal{N}^{\mu \nu \rho \sigma} h_{\mu \nu} f_{\rho \sigma}\right),
\end{aligned}
$$

where the coefficients $\mathcal{K}_{\Phi}, \mathcal{G}, \mathcal{N}$, and $\mathcal{M}_{\Phi}$ consist of all the possible combinations with the Minkowski metric $\eta_{\mu \nu}$,

$$
\begin{aligned}
\mathcal{K}_{\Phi}^{\alpha \beta \mid \mu \nu \rho \sigma}= & \kappa_{\Phi 1} \eta^{\alpha \beta} \eta^{\mu \rho} \eta^{\nu \sigma}+\kappa_{\Phi 2} \eta^{\mu \alpha} \eta^{\rho \beta} \eta^{\nu \sigma}+\kappa_{\Phi 3} \eta^{\alpha \mu} \eta^{\nu \beta} \eta^{\rho \sigma} \\
+ & \kappa_{\Phi 4} \eta^{\alpha \beta} \eta^{\mu \nu} \eta^{\rho \sigma} \\
\mathcal{G}^{\alpha \beta \mid \mu \nu \rho \sigma}= & \left(l_{1} \eta^{\alpha \beta} \eta^{\mu \rho}+l_{2} \eta^{\mu \alpha} \eta^{\rho \beta}\right) \eta^{\nu \sigma}+\left(l_{3} \eta^{\alpha \mu} \eta^{\nu \beta}\right. \\
& \left.+l_{4} \eta^{\alpha \beta} \eta^{\mu \nu}\right) \eta^{\rho \sigma}+l_{5} \eta^{\mu \nu} \eta^{\beta \sigma} \eta^{\alpha \rho} \\
& \mathcal{M}_{\Phi}^{\mu \nu \rho \sigma}=\mu_{\Phi 1} \eta^{\mu \rho} \eta^{\nu \sigma}+\mu_{\Phi 2} \eta^{\mu \nu} \eta^{\rho \sigma} \\
& \mathcal{N}^{\mu \nu \rho \sigma}=n_{1} \eta^{\mu \rho} \eta^{\nu \sigma}+n_{2} \eta^{\mu \nu} \eta^{\rho \sigma}
\end{aligned}
$$

\footnotetext{
${ }^{1}$ Strictly speaking, one can also include a Lorentz-invariant scalar $\eta_{\mu \nu} x^{\mu} x^{\nu}=-t^{2}+\mathbf{x}^{2}$ for theories invariant under a global Lorentz transformation. Once introducing this scalar quantity, the analysis would be more complicated. For simplicity, we here do not consider such a possibility.
} 
and we defined the label $\Phi=(h, f)$. A comma denotes a par tial derivative with respect to coordinates. Here, $\kappa_{\Phi 1, \Phi 2, \Phi 3, \Phi 4}$, $l_{1,2,3,4,5}, \mu_{\Phi 1, \Phi 2}$, and $n_{1,2}$ are constant parameters. The linearized Hassan-Rosen bigravity corresponds to

$$
\begin{aligned}
\kappa_{h 2} & =-\kappa_{h 3}=2 \kappa_{h 4}=-2 \kappa_{h 1}, \\
\kappa_{f 2} & =-\kappa_{f 3}=2 \kappa_{f 4}=-2 \kappa_{f 1}, \\
l_{1} & =l_{2}=l_{3}=l_{4}=l_{5}=0, \\
\mu_{h 2} & =-\mu_{h 1}, \quad \mu_{f 2}=-\mu_{f 1}, \\
n_{2} & =-n_{1}=2 \sqrt{\mu_{h 1} \mu_{f 1}},
\end{aligned}
$$

as shown in (2), and this theory is invariant under the gauge transformation

$$
\begin{gathered}
h_{\mu \nu} \rightarrow h_{\mu \nu}+\frac{1}{2 \sqrt{\mu_{h 1}}}\left(\partial_{\mu} \xi_{\nu}+\partial_{\nu} \xi_{\mu}\right), \\
f_{\mu \nu} \rightarrow f_{\mu \nu}+\frac{1}{2 \sqrt{\mu_{f 1}}}\left(\partial_{\mu} \xi_{\nu}+\partial_{\nu} \xi_{\mu}\right) .
\end{gathered}
$$

Alternatively, one can diagonalize the mass terms to remove $n_{1}$ and $n_{2}$ without changing the kinetic terms by taking linear combinations of $h_{\mu \nu}$ and $f_{\mu \nu}$, and then the resultant theory satisfies

$$
\begin{aligned}
\kappa_{h 2} & =-\kappa_{h 3}=2 \kappa_{h 4}=-2 \kappa_{h 1}, \\
\kappa_{f 2} & =-\kappa_{f 3}=2 \kappa_{f 4}=-2 \kappa_{f 1}, \\
\mu_{h 2} & =-\mu_{h 1}, \quad \mu_{f 1}=\mu_{f 2}=0, \\
l_{1} & =l_{2}=l_{3}=l_{4}=l_{5}=n_{1}=n_{2}=0,
\end{aligned}
$$

as found in (4). Then this theory with (12) is invariant under the gauge transformation

$$
h_{\mu \nu} \rightarrow h_{\mu \nu}, \quad f_{\mu \nu} \rightarrow f_{\mu \nu}+\partial_{\mu} \xi_{\nu}+\partial_{\nu} \xi_{\mu} .
$$

Thus, this is nothing but the Fierz-Pauli massive spin-2 field for $h_{\mu \nu}$ and the linearized general relativity for $f_{\mu \nu}$.

\section{B. Scalar-vector-tensor decomposition}

Following [21], we decompose the rank-2 symmetric tensor fields $h_{\mu \nu}$ and $f_{\mu \nu}$ into transverse-traceless tensors, transverse vectors, and scalars as

$$
\begin{gathered}
\Phi_{00}=\Phi^{00}=-2 \alpha_{\Phi}, \\
\Phi_{0 i}=-\Phi^{0 i}=\beta_{, i}^{\Phi}+B_{i}^{\Phi} \quad\left(B_{\Phi, i}^{i}=0\right), \\
\Phi_{i j}=\Phi^{i j}=2 \mathcal{R}_{\Phi} \delta_{i j}+2 \mathcal{E}_{, i j}^{\Phi}+F_{i, j}^{\Phi}+F_{j, i}^{\Phi}+2 H_{i j}^{\Phi} \\
\left(F_{\Phi, i}^{i}=0, \quad H_{\Phi i}^{i}=H_{\Phi, j}^{i j}=0\right) .
\end{gathered}
$$

Here, scalar, vector, and tensors are defined based on transformation properties with respect to a three-dimensional rotation in Minkowski spacetime, and the transverse-traceless tensors $H_{i j}^{\Phi}$, two transverse vectors $B_{i}^{\Phi}$ and $F_{i}^{\Phi}$, and four scalars $\alpha_{\Phi}, \beta_{\Phi}, \mathcal{R}_{\Phi}$, and $\mathcal{E}_{\Phi}$, respectively, have two, four, and four components in each $\Phi$. Since we focus on the ories with $7 \mathrm{DOFs}$, to be more precise $2 \times 2$ (tensor) +2 (vector) +1 (scalar) DOFs, we need to eliminate six components of the transverse vectors and seven components of the scalars, and then the final DOFs become $20-6-7=$ 7 DOFs. Under this decomposition, the quadratic action can always be separated into three parts, which solely consists of scalar, vector, and tensor perturbations, respectively,

$S\left[h_{\mu \nu}, f_{\mu \nu}\right]=S^{S}\left[\alpha_{\Phi}, \beta_{\Phi}, \mathcal{R}_{\Phi}, \mathcal{E}_{\Phi}\right]+S^{V}\left[B_{i}^{\Phi}, F_{i}^{\Phi}\right]+S^{T}\left[H_{i j}^{\Phi}\right]$.

In the following section, we will examine each sector and derive conditions to have theories with 7 DOFs by the Hamiltonian analysis. Hereafter, we replace all the spatial derivatives as $\partial^{2} \rightarrow-k^{2}$ after integrating by parts, where $k$ is the wave number in the Fourier space.

\section{TENSOR SECTOR}

The action in the tensor sector is found to be

$$
\begin{aligned}
S^{T}\left[H_{i j}^{h}, H_{i j}^{f}\right]= & 4 \int d t d^{3} k\left[\kappa_{h 1}\left(\dot{H}_{i j}^{h}\right)^{2}-\left(\kappa_{h 1} k^{2}+\mu_{h 1}\right)\left(H_{i j}^{h}\right)^{2}\right. \\
& +\kappa_{f 1}\left(\dot{H}_{i j}^{f}\right)^{2}-\left(\kappa_{f 1} k^{2}+\mu_{f 1}\right)\left(H_{i j}^{f}\right)^{2} \\
& \left.-l_{1} \dot{H}_{i j}^{h} \dot{H}_{f}^{i j}-\left(k^{2} l_{1}+n_{1}\right) H_{i j}^{h} H_{f}^{i j}\right],
\end{aligned}
$$

where a dot denotes the time derivative. It is manifest that the action is symmetric under the replacements $h$ and $f$, and hence the result will be applied to both modes in parallel. Throughout this paper, assuming $\kappa_{h 1} \neq 0$, we set

$$
l_{1}=0,
$$

which can be achieved by a field redefinition of $f$ without loss of generality (see Appendix A). Thanks to $l_{1}=0$ by the field redefinition, the kinetic matrix composed of $h$ and $f$ is diagonal, and the existence and ghost-free conditions of both the tensor modes require

$$
\kappa_{h 1}>0, \quad \kappa_{f 1}>0
$$

Hereafter, we impose the condition (19), and it is manifest that the physical degrees of freedom in the tensor sector is 2 for each field.

\section{VECTOR SECTOR}

In this section, we perform the Hamiltonian analysis for the vector variables. In order to have a theory with 7 DOFs in total, the vector sector should have 2 physical DOFs, which means the reduction of the phase space is necessary 
in the view point of the Hamiltonian analysis. We first rescale $F_{i}^{\Phi}$ as $F_{i}^{\Phi} \rightarrow F_{i}^{\Phi} / k$ for convenience. Then, the action in the vector sector is given by

$$
S^{V}\left[B_{i}^{\Phi}, F_{i}^{\Phi}\right]=\int d t d^{3} k\left(\mathcal{L}_{\text {kin }}^{V}+\mathcal{L}_{\text {cross }}^{V}+\mathcal{L}_{\text {mass }}^{V}\right)
$$

where each Lagrangian is given by

$$
\begin{aligned}
\mathcal{L}_{\text {kin }}^{V}= & -\left(2 \kappa_{h 1}+\kappa_{h 2}\right)\left(\dot{B}_{i}^{h}\right)^{2}+2 \kappa_{h 1}\left(\dot{F}_{i}^{h}\right)^{2} \\
& -\left(2 \kappa_{f 1}+\kappa_{f 2}\right)\left(\dot{B}_{i}^{f}\right)^{2}+2 \kappa_{f 1}\left(\dot{F}_{i}^{f}\right)^{2}-l_{2} \dot{B}_{i}^{h} \dot{B}_{i}^{f},
\end{aligned}
$$

$$
\left(\begin{array}{c}
\pi_{B_{i}^{h}} \\
\pi_{B_{i}^{f}} \\
\pi_{F_{i}^{h}}^{h} \\
\pi_{F_{i}^{f}}
\end{array}\right)=\left(\begin{array}{ccc}
-2\left(2 \kappa_{h 1}+\kappa_{h 2}\right) & -l_{2} & 0 \\
-l_{2} & -2\left(2 \kappa_{f 1}+\kappa_{f 2}\right) & 0 \\
0 & 0 & 4 \kappa_{h 1} \\
0 & 0 & 0
\end{array}\right.
$$

Then the Hamiltonian is defined by

$$
\mathcal{H}^{V}=\dot{B}_{i}^{h} \pi_{B_{i}^{h}}+\dot{B}_{i}^{f} \pi_{B_{i}^{f}}+\dot{F}_{i}^{h} \pi_{F_{i}^{h}}+\dot{F}_{i}^{f} \pi_{F_{i}^{f}}-\mathcal{L}^{V} .
$$

As one can see from (21), the kinetic parts of $B_{i}^{\Phi}$ and $F_{i}^{\Phi}$ are completely decoupled; i.e., the kinetic matrix is block diagonalized and the kinetic terms for $F_{i}^{\Phi}$ indeed exist since we have imposed (19). Therefore, in order to see the degeneracy of the vector sector, it allows us to consider only the kinetic matrix of $B_{i}^{\Phi}$, which is

$$
\mathcal{K}_{V}=\left(\begin{array}{cc}
-2\left(2 \kappa_{h 1}+\kappa_{h 2}\right) & -l_{2} \\
-l_{2} & -2\left(2 \kappa_{f 1}+\kappa_{f 2}\right)
\end{array}\right) .
$$

$$
\begin{aligned}
\mathcal{L}_{\text {mass }}^{V} & =2\left(k^{2} \kappa_{h 1}+\mu_{h 1}\right)\left(B_{i}^{h}\right)^{2}-\left(k^{2}\left(2 \kappa_{h 1}+\kappa_{h 2}\right)+2 \mu_{h 1}\right)\left(F_{i}^{h}\right)^{2} \\
& +2\left(k^{2} \kappa_{f 1}+\mu_{f 1}\right)\left(B_{i}^{f}\right)^{2}-\left(k^{2}\left(2 \kappa_{f 1}+\kappa_{f 2}\right)+2 \mu_{f 1}\right)\left(F_{i}^{f}\right)^{2} \\
& +2 n_{1} B_{i}^{h} B_{i}^{f}-\left(k^{2} l_{2}+2 n_{1}\right) F_{i}^{h} F_{i}^{f} .
\end{aligned}
$$

The relation between conjugate momentum $\pi_{\Phi} \equiv \partial \mathcal{L} / \partial \dot{\Phi}$ and the time derivatives of canonical variables of $B_{i}^{\Phi}$ and $F_{i}^{\Phi}$ is found to be

$$
\mathcal{L}_{\text {cross }}^{V}=2 k \kappa_{h 2} B_{i}^{h} \dot{F}_{i}^{h}+2 k \kappa_{f 2} B_{i}^{h} \dot{F}_{i}^{f}+k l_{2}\left(B_{i}^{h} \dot{F}_{i}^{f}+B_{i}^{f} \dot{F}_{i}^{h}\right),
$$

$$
\left.\begin{array}{c}
0 \\
0 \\
4 \kappa_{f 1}
\end{array}\right)\left(\begin{array}{c}
\dot{B}_{i}^{h} \\
\dot{B}_{i}^{f} \\
\dot{F}_{i}^{h} \\
\dot{F}_{i}^{f}
\end{array}\right)+\left(\begin{array}{cccc}
0 & 0 & 0 & 0 \\
0 & 0 & 0 & 0 \\
2 k \kappa_{h 2} & k l_{2} & 0 & 0 \\
k l_{2} & 2 k \kappa_{f 2} & 0 & 0
\end{array}\right)\left(\begin{array}{c}
B_{i}^{h} \\
B_{i}^{f} \\
F_{i}^{h} \\
F_{i}^{f}
\end{array}\right) .
$$

The eigenvalue equation $\mathcal{F}_{V}(\lambda)$ of the kinetic matrix $\mathcal{K}_{V}$ is found to be

$$
\begin{aligned}
\mathcal{F}_{V}(\lambda) & \equiv \operatorname{det}\left(\mathcal{K}_{V}-\lambda I\right) \\
& =\left(4 \kappa_{f 1}+2 \kappa_{f 2}+\lambda\right)\left(4 \kappa_{h 1}+2 \kappa_{h 2}+\lambda\right)-l_{2}^{2} \\
& =0 .
\end{aligned}
$$

The determinant of the kinetic matrix is simply given by $\operatorname{det} \mathcal{K}_{V}=\mathcal{F}_{V}(0)$.

Now we would like to classify the cases based on the number of primary constraints as follows:

$$
\begin{gathered}
2 \text { primary constraints }: \mathcal{F}_{V}(0)=0 \quad \& \quad \mathcal{F}_{V}^{\prime}(0) \neq 0 \leftrightarrow \kappa_{f 2}=-2 \kappa_{f 1}+\frac{l_{2}^{2}}{4\left(2 \kappa_{h 1}+\kappa_{h 2}\right)}, \\
4 \text { primary constraints }: \mathcal{F}_{V}(0)=0 \& \mathcal{F}_{V}^{\prime}(0)=0 \leftrightarrow \kappa_{h 2}=-2 \kappa_{h 1} \& \kappa_{f 2}=-2 \kappa_{f 1} \quad \& \quad l_{2}=0 .
\end{gathered}
$$

Here, at this point, $h$ and $f$ are symmetric; therefore, the case with $2 \kappa_{h 1}+\kappa_{h 2}=0$ in the case of two primary constraints can be obtained by simply replacing $h$ and $f$. When there are only two primary constraints, the Hamiltonian analysis shows that the number of the final physical DOFs can be at least 4 , and it is the undesired number. The analysis for two primary cases is summarized in Appendix C. For this reason, hereafter we only consider four4 primary cases, where both $B_{i}^{h}$ and $B_{i}^{f}$ become nondynamical.
In this case, we have four primary constraints, which are given by

$$
\begin{aligned}
& \mathcal{C}_{B_{i}^{h}}^{(1)} \equiv \pi_{B_{i}^{h}} \approx 0, \\
& \mathcal{C}_{B_{i}^{f}}^{(1)} \equiv \pi_{B_{i}^{f}} \approx 0,
\end{aligned}
$$

and we define the total Hamiltonian by adding the Lagrange multipliers $\lambda_{B_{i}^{h}}$ and $\lambda_{B_{i}^{f}}$, 


$$
\mathcal{H}_{T}^{V}=\mathcal{H}^{V}+\lambda_{B_{i}^{h}} \mathcal{C}_{B_{i}^{h}}^{(1)}+\lambda_{B_{i}^{f}} \mathcal{C}_{B_{i}^{f}}^{(1)}
$$

Then the time evolution of the primary constraints generates the secondary constraints

$$
\begin{gathered}
\mathcal{C}_{B_{i}^{h}}^{(2)} \equiv\left\{\mathcal{C}_{B_{i}^{h}}^{(2)}, \mathcal{H}_{T}^{V}\right\}=4 \mu_{h 1} B_{i}^{h}+2 n_{1} B_{i}^{f}-k \pi_{F_{i}^{h}} \approx 0, \\
\mathcal{C}_{B_{i}^{f}}^{(2)} \equiv\left\{\mathcal{C}_{B_{i}^{f}}^{(2)}, \mathcal{H}_{T}^{V}\right\}=2 n_{1} B_{i}^{h}+4 \mu_{f 1} B_{i}^{f}-k \pi_{F_{i}^{f}} \approx 0,
\end{gathered}
$$

and the time evolution of the secondary constraints gives

$$
\begin{aligned}
\left(\begin{array}{c}
\dot{\mathcal{C}}_{B_{i}^{(2)}}^{(2)} \\
\dot{\mathcal{C}}_{B_{i}^{f}}^{(2)}
\end{array}\right) & =\left(\begin{array}{c}
\left\{\mathcal{C}_{B_{i}^{h}}^{(2)}, \mathcal{H}_{T}^{V}\right\} \\
\left\{\mathcal{C}_{B_{i}^{f}}^{(2)}, \mathcal{H}_{T}^{V}\right\}
\end{array}\right) \\
& =\left(\begin{array}{c}
\left\{\mathcal{C}_{B_{i}^{h}}^{(2)}, \mathcal{H}^{V}\right\} \\
\left\{\mathcal{C}_{B_{i}^{f}}^{(2)}, \mathcal{H}^{V}\right\}
\end{array}\right)+\left(\begin{array}{cc}
4 \mu_{h 1} & 2 n_{1} \\
2 n_{1} & 4 \mu_{f 1}
\end{array}\right)\left(\begin{array}{c}
\lambda_{B_{i}^{h}} \\
\lambda_{B_{i}^{f}}
\end{array}\right) \approx 0 .
\end{aligned}
$$

Therefore, when $n_{1}^{2}-4 \mu_{f 1} \mu_{h 1} \neq 0$, namely the coefficient matrix in front of the Lagrange multipliers is not degenerate, all the Lagrange multipliers $\lambda_{B_{i}^{h}}$ and $\lambda_{B_{i}^{f}}$ are determined by the above equations, and all the primary and secondary constraints are second class. In this case, the total number of physical DOFs is $(8 \times 2-8) / 2=4$, and thus we disregard this option.

On the other hand, when $n_{1}^{2}-4 \mu_{f 1} \mu_{h 1}=0$, the coefficient matrix in front of the Lagrange multipliers is degenerate, and two out of four Lagrange multip liers cannot be determined. Hereafter we assume $\mu_{h 1} \neq$ 0 and solve $n_{1}^{2}-4 \mu_{f 1} \mu_{h 1}=0$ for $\mu_{f 1}{ }^{2}$ It is convenient to redefine the primary constraints associated with $B_{i}^{f}$ as a linear combination of the original primary constraints:

$$
\tilde{\mathcal{C}}_{B_{i}^{h}}^{(1)} \equiv \pi_{B_{i}^{h}} \approx 0
$$

$$
\tilde{\mathcal{C}}_{B_{i}^{f}}^{(1)} \equiv \pi_{B_{i}^{f}}-\frac{n_{1}}{2 \mu_{h 1}} \pi_{B_{i}^{h}} \approx 0 .
$$

We also redefine the total Hamiltonian

$$
\mathcal{H}_{T}^{V}=\mathcal{H}^{V}+\tilde{\lambda}_{B_{i}^{h}} \tilde{\mathcal{C}}_{B_{i}^{h}}^{(1)}+\tilde{\lambda}_{B_{i}^{f}} \tilde{\mathcal{C}}_{B_{i}^{f}}^{(1)}
$$

Then the secondary constraints become

$$
\begin{aligned}
& \tilde{\mathcal{C}}_{B_{i}^{h}}^{(2)} \equiv\left\{\tilde{\mathcal{C}}_{B_{i}^{h}}^{(1)}, \mathcal{H}_{T}^{V}\right\}=4 \mu_{h 1} B_{i}^{h}+2 n_{1} B_{i}^{f}-k \pi_{F_{i}^{h}} \approx 0, \\
& \tilde{\mathcal{C}}_{B_{i}^{f}}^{(2)} \equiv\left\{\tilde{\mathcal{C}}_{B_{i}^{f}}^{(1)}, \mathcal{H}_{T}^{V}\right\}=k\left(\frac{n_{1}}{2 \mu_{h 1}} \pi_{F_{i}^{h}}-\pi_{F_{i}^{f}}\right) \approx 0 .
\end{aligned}
$$

The time evolution of the secondary constraints yields

$$
\begin{gathered}
\dot{\tilde{\mathcal{C}}}_{B_{i}^{h}}^{(2)}=\left\{\tilde{\mathcal{C}}_{B_{i}^{h}}^{(2)}, \mathcal{H}_{T}^{V}\right\}=2 k\left(2 \mu_{h 1} F_{i}^{h}+n_{1} F_{i}^{f}\right)+4 \mu_{h 1} \tilde{\lambda}_{B_{i}^{h}} \approx 0, \\
\dot{\tilde{\mathcal{C}}}_{B_{i}^{f}}^{(2)}=\left\{\tilde{\mathcal{C}}_{B_{i}^{f}}^{(2)}, \mathcal{H}_{T}^{V}\right\} \approx 0 .
\end{gathered}
$$

Here, the time evolution of the secondary constraints $\tilde{\mathcal{C}}_{B_{i}^{f}}^{(2)}$ is trivially zero. Therefore, two of the Lagrange multipliers can be determined by the time evolution of the secondary constraints, and the rest of them are undetermined. Since the constraints $\tilde{\mathcal{C}}_{B_{i}^{f}}^{(1)}$ and $\tilde{\mathcal{C}}_{B_{i}^{f}}^{(2)}$ commute with all the constraints including themselves, they are firstclass constraints. To summarize, we find

$$
\text { Vector DOF }=\frac{8 \times 2-4(2 \text { primary } \& 2 \text { secondary })-4(2 \text { primary } \& 2 \text { secondary }) \times 2 \text { (first-class })}{2}=2
$$

The choice of the coefficients are

$$
\begin{gathered}
\kappa_{h 2}=-2 \kappa_{h 1} \neq 0, \quad \kappa_{f 2}=-2 \kappa_{f 1} \neq 0, \\
l_{2}=0, \quad \mu_{f 1}=\frac{n_{1}^{2}}{4 \mu_{h 1}}, \quad \mu_{h 1} \neq 0 .
\end{gathered}
$$

\footnotetext{
${ }^{2}$ For the $\mu_{h 1}=0$ case, one can simply switch all the notation of $h$ and $f$. When $\mu_{h 1}=\mu_{f 1}=n_{1}=0$, all the constraints become first class, implying that the physical degrees of freedom are zero in the vector sector.
}

In the analysis for the scalar sector in the next section, conditions (19) and (44) are imposed.

\section{SCALAR SECTOR}

In this section, we focus on the scalar sector. Here, we need to eliminate 7 DOFs in the scalar sector in order to have 1 physical DOF. Introducing dimensionless variables, $\beta_{\Phi} \rightarrow \beta_{\Phi} / k$ and $\mathcal{E}_{\Phi} \rightarrow \mathcal{E}_{\Phi} / k^{2}$, the Lagrangian reduces to

$$
\mathcal{L}^{S}=\mathcal{L}_{\text {kin }}^{S}+\mathcal{L}_{\text {cross }}^{S}+\mathcal{L}_{\text {mass }}^{S}
$$


where the explicit form of the first part reads

$$
\begin{aligned}
\mathcal{L}_{\text {kin }}^{S}= & 4\left(\kappa_{h 1}+\kappa_{h 2}+\kappa_{h 3}+\kappa_{h 4}\right) \dot{\alpha}_{h}^{2}-\left(2 \kappa_{h 1}+\kappa_{h 2}\right) \dot{\beta}_{h}^{2}+12\left(\kappa_{h 1}+3 \kappa_{h 4}\right) \dot{\mathcal{R}}_{h}^{2}+4\left(\kappa_{h 1}+\kappa_{h 4}\right) \dot{\mathcal{E}}_{h}^{2} \\
& -4\left(\kappa_{h 3}+2 \kappa_{h 4}\right)\left(-3 \dot{\mathcal{R}}_{h}+\dot{\mathcal{E}}_{h}\right) \dot{\alpha}_{h}-8\left(\kappa_{h 1}+3 \kappa_{h 4}\right) \dot{\mathcal{R}}_{h} \dot{\mathcal{E}}_{h}+4\left(\kappa_{f 1}+\kappa_{f 2}+\kappa_{f 3}+\kappa_{f 4}\right) \dot{\alpha}_{f}^{2}-\left(2 \kappa_{f 1}+\kappa_{f 2}\right) \dot{\beta}_{f}^{2} \\
& +12\left(\kappa_{f 1}+3 \kappa_{f 4}\right) \dot{\mathcal{R}}_{f}^{2}+4\left(\kappa_{f 1}+\kappa_{f 4}\right) \dot{\mathcal{E}}_{f}^{2}-4\left(\kappa_{f 3}+2 \kappa_{f 4}\right)\left(-3 \dot{\mathcal{R}}_{f}+\dot{\mathcal{E}}_{f}\right) \dot{\alpha}_{f}-8\left(\kappa_{f 1}+3 \kappa_{f 4}\right) \dot{\mathcal{R}}_{f} \dot{\mathcal{E}}_{f} \\
& +4\left(l_{2}+l_{3}+l_{4}+l_{5}\right) \dot{\alpha}_{h} \dot{\alpha}_{f}-l_{2} \dot{\beta}_{h} \dot{\beta}_{f}+36 l_{4} \dot{\mathcal{R}}_{h} \dot{\mathcal{R}}_{f}+4 l_{4} \dot{\mathcal{E}}_{h} \dot{\mathcal{E}}_{f}+12\left(l_{3}+l_{4}\right) \dot{\alpha}_{h} \dot{\mathcal{R}}_{f}+12\left(l_{4}+l_{5}\right) \dot{\alpha}_{f} \dot{\mathcal{R}}_{h} \\
& -4\left(l_{3}+l_{4}\right) \dot{\alpha}_{h} \dot{\mathcal{E}}_{f}-4\left(l_{4}+l_{5}\right) \dot{\alpha}_{f} \dot{\mathcal{E}}_{h}-12 l_{4}\left(\dot{\mathcal{R}}_{h} \dot{\mathcal{E}}_{f}+\dot{\mathcal{R}}_{f} \dot{\mathcal{E}}_{h}\right),
\end{aligned}
$$

and the explicit expression for the remaining parts can be found in Appendix B. Once we impose the condition (44), the time derivative of $\beta_{h}$ and $\beta_{f}$ vanishes in the Lagrangian; hence $\beta_{h}$ and $\beta_{f}$ can be treated as the nondynamical variables. By utilizing the field redefinition summarized in Appendix A, we can further impose without loss of generality

$\kappa_{h 3}=2 \kappa_{h 1}, \quad \kappa_{f 3}=2 \kappa_{f 1}, \quad l_{3}+l_{4}+l_{5}=0$,

in addition to $l_{1}=0$. Hereafter, we assume these conditions to simplify the discussion. The conjugate momenta can be written as

$$
\left(\begin{array}{c}
\pi_{\alpha_{h}} \\
\pi_{\mathcal{R}_{h}} \\
\pi_{\mathcal{E}_{h}} \\
\pi_{\alpha_{f}} \\
\pi_{\mathcal{R}_{f}} \\
\pi_{\mathcal{E}_{f}}
\end{array}\right)=4 \mathcal{K}_{S}\left(\begin{array}{c}
\dot{\alpha}_{h} \\
\dot{\mathcal{R}}_{h} \\
\dot{\mathcal{E}}_{h} \\
\dot{\alpha}_{f} \\
\dot{\mathcal{R}}_{f} \\
\dot{\mathcal{E}}_{f}
\end{array}\right)+4\left(\begin{array}{c}
0 \\
-4 \kappa_{h 1} \\
0 \\
-l_{3} \\
-3 l_{3} \\
l_{3}
\end{array}\right) k \beta_{h}+4\left(\begin{array}{c}
-l_{5} \\
-3 l_{5} \\
l_{5} \\
0 \\
-4 \kappa_{f 1} \\
0
\end{array}\right) k \beta_{f}
$$

where the kinetic matrix for the scalar variables $\left\{\alpha_{h}, \mathcal{R}_{h}, \mathcal{E}_{h}, \alpha_{f}, \mathcal{R}_{f}, \mathcal{E}_{f}\right\}$ is given by

$$
\mathcal{K}_{S}=\left(\begin{array}{cccccc}
2\left(\kappa_{h 1}+\kappa_{h 4}\right) & 6\left(\kappa_{h 1}+\kappa_{h 4}\right) & -2\left(\kappa_{h 1}+\kappa_{h 4}\right) & 0 & -3 l_{5} & l_{5} \\
* & 6\left(\kappa_{h 1}+3 \kappa_{h 4}\right) & -2\left(\kappa_{h 1}+3 \kappa_{h 4}\right) & -3 l_{3} & -9\left(l_{3}+l_{5}\right) & 3\left(l_{3}+l_{5}\right) \\
* & * & 2\left(\kappa_{h 1}+\kappa_{h 4}\right) & l_{3} & 3\left(l_{3}+l_{5}\right) & -\left(l_{3}+l_{5}\right) \\
* & * & * & 2\left(\kappa_{f 1}+\kappa_{f 4}\right) & 6\left(\kappa_{f 1}+\kappa_{f 4}\right) & -2\left(\kappa_{f 1}+\kappa_{f 4}\right) \\
* & * & * & * & 6\left(\kappa_{f 1}+3 \kappa_{f 4}\right) & -2\left(\kappa_{f 1}+3 \kappa_{f 4}\right) \\
* & * & * & * & * & 2\left(\kappa_{f 1}+\kappa_{f 4}\right)
\end{array}\right),
$$

and

$$
\mathcal{F}_{S}(\lambda) \equiv \operatorname{det}\left(\mathcal{K}_{S}-\lambda I\right)
$$

$$
\pi_{\beta_{h}}=\pi_{\beta_{f}}=0 .
$$

Note that there are at least two primary constraints from $\pi_{\beta_{h}}=0$ and $\pi_{\beta_{f}}=0$. The Hamiltonian is given by

$$
\begin{aligned}
\mathcal{H}^{S}= & \dot{\alpha}_{h} \pi_{\alpha_{h}}+\dot{\alpha}_{f} \pi_{\alpha_{f}}+\dot{\mathcal{R}}_{h} \pi_{\mathcal{R}_{h}}+\dot{\mathcal{R}}_{f} \pi_{\mathcal{R}_{f}}+\dot{\mathcal{E}}_{h} \pi_{\mathcal{E}_{h}} \\
& +\dot{\mathcal{E}}_{f} \pi_{\mathcal{E}_{f}}+\dot{\beta}_{h} \pi_{\beta_{h}}+\dot{\beta}_{f} \pi_{\beta_{f}}-\mathcal{L}^{S} .
\end{aligned}
$$

\section{A. Classification of primary constraints}

Now, we would like to classify the cases based on the number of primary constraints. As performed in the analysis of the vector sector, we consider the eigenvalue equation,

The eigenvalue equation with $\lambda=0$, namely the determinant of the kinetic matrix, reads

$$
\begin{aligned}
\operatorname{det} \mathcal{K}_{S}= & \mathcal{F}_{S}(0) \\
= & 16 \kappa_{h 1} \kappa_{f 1}\left[8\left(\kappa_{f 1}+\kappa_{f 4}\right) \kappa_{h 1}\right. \\
& \left.+3 l_{3}^{2}\right]\left[8\left(\kappa_{h 1}+\kappa_{h 4}\right) \kappa_{f 1}+3 l_{5}^{2}\right] .
\end{aligned}
$$

When the above determinant is nonzero, that is, $\operatorname{det} \mathcal{K}_{S} \neq 0$, there are only two primary constraints, which can be defined by (50). In this case, the number of the physical DOFs is 4 as proved in Appendix D. Therefore, we disregard this option. The case of three primary constraints can be obtained by demanding $\mathcal{F}_{S}(0)=0$, 
3 primary constraints : $\mathcal{F}_{S}(0)=0 \leftrightarrow \kappa_{f 4}=-\kappa_{f 1}-\frac{3 l_{3}^{2}}{8 \kappa_{h 1}} \quad$ or $\quad \kappa_{h 4}=-\kappa_{h 1}-\frac{3 l_{5}^{2}}{8 \kappa_{f 1}}$.

Using the conditions above, we have

$$
\mathcal{F}_{S}^{\prime}(0)= \begin{cases}-8 \kappa_{f 1}\left(5 l_{3}^{2}+8 \kappa_{h 1}^{2}\right)\left(3 l_{5}^{2}+8 \kappa_{f 1}\left(\kappa_{h 1}+\kappa_{h 4}\right)\right) & \text { for } \kappa_{f 4}=-\kappa_{f 1}-\frac{3 l_{3}^{2}}{8 \kappa_{h l}}, \\ -8 \kappa_{h 1}\left(5 l_{5}^{2}+8 \kappa_{f 1}^{2}\right)\left(3 l_{3}^{2}+8 \kappa_{h 1}\left(\kappa_{f 1}+\kappa_{f 4}\right)\right) & \text { for } \kappa_{h 4}=-\kappa_{h 1}-\frac{3 l_{5}^{2}}{8 \kappa_{f 1}} .\end{cases}
$$

Now, $\mathcal{F}_{S}^{\prime}(0)=0$ gives only one solution of four primary cases due to the symmetric property under $h$ and $f$ in (54),

4 primary constraints : $\mathcal{F}_{S}(0)=0 \& \mathcal{F}_{S}^{\prime}(0)=0$

$$
\leftrightarrow \kappa_{h 4}=-\kappa_{h 1}-\frac{3 l_{5}^{2}}{8 \kappa_{f 1}} \& \kappa_{f 4}=-\kappa_{f 1}-\frac{3 l_{3}^{2}}{8 \kappa_{h 1}} .
$$

The absence of the case with five primary constraints can be proved as follows. In addition to $\mathcal{F}_{S}(0)=0$ and $\mathcal{F}_{S}^{\prime}(0)=0$, we further need to impose $\mathcal{F}_{S}^{\prime \prime}(0)=0$, which is given by

$$
\begin{aligned}
\mathcal{F}_{S}^{\prime \prime}(0)= & 32\left(l_{5}^{2}+16 \kappa_{f 1}^{2}\right)\left(\kappa_{h 1}-\frac{9 l_{3} l_{5} \kappa_{f 1}}{l_{5}^{2}+16 \kappa_{f 1}^{2}}\right)^{2} \\
& +\frac{8 l_{3}^{2}\left(5 l_{5}^{2}+8 \kappa_{f 1}^{2}\right)^{2}}{l_{5}^{2}+16 \kappa_{f 1}^{2}}=0 .
\end{aligned}
$$

It is manifest that there is no real solution for this equation under the assumption (19), and therefore, the scalar sector cannot have five or more primary constraints.

\section{B. Three primary constraints}

In this subsection, we consider the case with three primary constraints. Although there are two options as in (54), they are essentially equivalent since they are transformed from each other as shown in Appendix A 2, which satisfies

$$
\begin{aligned}
& \kappa_{h 2}=-\kappa_{h 3}=-2 \kappa_{h 1} \neq 0, \quad \kappa_{f 2}=-\kappa_{f 3}=-2 \kappa_{f 1} \neq 0, \\
& \kappa_{f 4}=-\kappa_{f 1}-\frac{3 l_{3}^{2}}{8 \kappa_{h 1}}, \\
& l_{1}=l_{2}=l_{4}=\mu_{f 1}=n_{1}=0, \quad l_{5}=-l_{3} .
\end{aligned}
$$

In this case, we have three primary constraints, which are defined by

$$
\begin{aligned}
& \mathcal{C}_{\alpha_{f}}^{(1)} \equiv \pi_{\alpha_{f}}-\frac{l_{3}}{4 \kappa_{h 1}}\left(\pi_{\mathcal{R}_{h}}-3 \pi_{\alpha_{h}}\right) \approx 0, \quad \mathcal{C}_{\beta_{h}}^{(1)} \equiv \pi_{\beta_{h}} \approx 0, \\
& \mathcal{C}_{\beta_{f}}^{(1)} \equiv \pi_{\beta_{f}} \approx 0 .
\end{aligned}
$$

The total Hamiltonian is given by

$$
\mathcal{H}_{T}^{S}=\mathcal{H}^{S}+\lambda_{\alpha_{f}} \mathcal{C}_{\alpha_{f}}^{(1)}+\lambda_{\beta_{h}} \mathcal{C}_{\beta_{h}}^{(1)}+\lambda_{\beta_{f}} \mathcal{C}_{\beta_{f}}^{(1)}
$$

The evolution of the primary constraints yields the secondary constraints

$$
\begin{aligned}
\mathcal{C}_{\alpha_{f}}^{(2)} \equiv & \left\{\mathcal{C}_{\alpha_{f}}^{(1)}, \mathcal{H}_{T}^{S}\right\}=-2\left(2 k^{2} l_{3}+2 n_{2}+\frac{3 l_{3} \mu_{h 1}}{\kappa_{h 1}}\right) \alpha_{h}+2\left(2 k^{2} l_{3}-6 n_{2}+\frac{3 l_{3} \mu_{h 1}}{\kappa_{h 1}}\right) \mathcal{R}_{h}+2\left(2 k^{2} l_{3}+2 n_{2}-\frac{l_{3} \mu_{h 1}}{\kappa_{h 1}}\right) \mathcal{E}_{h} \\
& +4\left(\frac{k^{2} l_{3}^{2}}{\kappa_{h 1}}-2 \mu_{f 2}\right)\left(\alpha_{f}-\mathcal{E}_{f}\right)+4\left(\frac{k^{2}\left(3 l_{3}^{2}+4 \kappa_{f 1} \kappa_{h 1}\right)}{\kappa_{h 1}}-6 \mu_{f 2}\right) \mathcal{R}_{f} \approx 0,
\end{aligned}
$$

$$
\begin{gathered}
\mathcal{C}_{\beta_{h}}^{(2)} \equiv\left\{\mathcal{C}_{\beta_{h}}^{(1)}, \mathcal{H}_{T}^{S}\right\}=-k\left(\pi_{\alpha_{h}}+\pi_{\mathcal{E}_{h}}\right)+4 \mu_{h 1} \beta_{h} \approx 0, \\
\mathcal{C}_{\beta_{f}}^{(2)} \equiv\left\{\mathcal{C}_{\beta_{f}}^{(1)}, \mathcal{H}_{T}^{S}\right\}=-k \pi_{\mathcal{E}_{f}}+\frac{k l_{3}}{4 \kappa_{h 1}}\left(3 \pi_{\alpha_{h}}-\pi_{\mathcal{R}_{h}}\right) \approx 0 .
\end{gathered}
$$

Here, none of the above constraints can be trivially zero with any choice of the parameters under the assumption (19). Then, the time evolution of the secondary constraint
$\mathcal{C}_{\beta_{f}}^{(2)}$ gives the tertiary constraint

$$
\begin{aligned}
\mathcal{C}_{\beta_{f}}^{(3)} \equiv & \left\{\mathcal{C}_{\beta_{f}}^{(2)}, \mathcal{H}_{T}^{S}\right\}=k \mathcal{C}_{\alpha_{f}}^{(2)}+4 k^{3}\left[l_{3}\left(\alpha_{h}-\mathcal{E}_{h}+3 \mathcal{R}_{h}\right)\right. \\
& \left.-4 \kappa_{f 1} \mathcal{R}_{f}\right] \approx 0,
\end{aligned}
$$

and its time evolution demands

$$
\dot{\mathcal{C}}_{\beta_{f}}^{(3)}=k\left(\dot{\mathcal{C}}_{\alpha_{f}}^{(2)}+k \mathcal{C}_{\beta_{f}}^{(2)}\right) \approx 0 .
$$


Since $\dot{\mathcal{C}}_{\beta_{f}}^{(3)}$ does not generate an independent equation, there is no more constraint from $\beta_{f}$. The evolution of the rest of the secondary constraints are given by

$$
\begin{aligned}
\left(\begin{array}{c}
\dot{\mathcal{C}}_{\alpha_{f}}^{(2)} \\
\dot{\mathcal{C}}_{\beta_{h}}^{(2)}
\end{array}\right)= & \left(\begin{array}{c}
\left\{\mathcal{C}_{\alpha_{f}}^{(2)}, \mathcal{H}_{T}^{S}\right\} \\
\left\{\mathcal{C}_{\beta_{h}}^{(2)}, \mathcal{H}_{T}^{S}\right\}
\end{array}\right)=\left(\begin{array}{c}
\left\{\mathcal{C}_{\alpha_{f}}^{(2)}, \mathcal{H}^{S}\right\} \\
\left\{\mathcal{C}_{\beta_{h}}^{(2)}, \mathcal{H}^{S}\right\}
\end{array}\right) \\
& +2\left(\begin{array}{cc}
-4 \mu_{f 2}-\frac{3 l_{3}^{2} \mu_{h 1}}{\kappa_{h 1}^{2}} & 0 \\
0 & 2 \mu_{h 1}
\end{array}\right)\left(\begin{array}{c}
\lambda_{\alpha_{f}} \\
\lambda_{\beta_{h}}
\end{array}\right) \approx 0 .
\end{aligned}
$$

Since $\mu_{h 1} \neq 0$, the Lagrange multiplier $\lambda_{\beta_{h}}$ is determined by $\dot{\mathcal{C}}_{\beta_{h}}^{(2)}=0$. When the Poisson bracket $\left\{\mathcal{C}_{\alpha_{f}}^{(2)}, \mathcal{C}_{\alpha_{f}}^{(1)}\right\}$, i.e., the coefficient of $\lambda_{\alpha_{f}}$ in $\dot{\mathcal{C}}_{\alpha_{f}}^{(2)}$, is nonvanishing, the evolution of $\mathcal{C}_{\alpha_{f}}^{(2)}$ determines the Lagrange multiplier $\lambda_{\alpha_{f}}$ and no more constraint from $\mathcal{C}_{\alpha_{f}}^{(2)}$ will be generated. Now we redefine the following constraints:

$\tilde{\mathcal{C}}_{\beta_{f}}^{(2)} \equiv \mathcal{C}_{\beta_{f}}^{(2)}-k \mathcal{C}_{\alpha_{f}}^{(1)} \approx 0, \quad \tilde{\mathcal{C}}_{\beta_{f}}^{(3)} \equiv \mathcal{C}_{\beta_{f}}^{(3)}-k \mathcal{C}_{\alpha_{f}}^{(2)} \approx 0$.

The constraints $\mathcal{C}_{\beta_{f}}^{(1)}$ and $\tilde{\mathcal{C}}_{\beta_{f}}^{(2,3)}$ commute with all constraints; therefore, these are first class. The rest of the constraints are second class. In summary, the number of the physical DOFs is $(8 \times 2-4-3 \times 2) / 2=3$.

In order to eliminate extra DOFs, one has to impose an extra condition $\left\{\mathcal{C}_{\alpha_{f}}^{(2)}, \mathcal{C}_{\alpha_{f}}^{(1)}\right\}=0$, namely

$$
\mu_{f 2}=-\frac{3 l_{3}^{2} \mu_{h 1}}{4 \kappa_{h 1}^{2}} .
$$

In this case, the evolution of $\mathcal{C}_{\alpha_{f}}^{(2)}$ yields the tertiary constraint when $l_{3} \neq 0$ or $n_{2} \neq 0$,

$$
\mathcal{C}_{\alpha_{f}}^{(3)} \equiv\left\{\mathcal{C}_{\alpha_{f}}^{(2)}, \mathcal{H}_{T}^{S}\right\} \approx 0
$$

where the explicit expression of $\mathcal{C}_{\alpha_{f}}^{(3)}$ is given in Appendix E. When $l_{3}=n_{2}=0, \mathcal{C}_{\alpha_{f}}^{(3)}=-k \mathcal{C}_{\beta_{f}}^{(2)}$, implying that there is no more constraint. Therefore, we, hereafter, consider the case with $l_{3} \neq 0$ or $n_{2} \neq 0$. Now, the evolution of $\mathcal{C}_{\alpha_{f}}^{(3)}$ yields the quaternary constraint $\mathcal{C}_{\alpha_{f}}^{(4)}=\left\{\mathcal{C}_{\alpha_{f}}^{(3)}, \mathcal{H}_{T}^{S}\right\} \approx 0$. Since $\mathcal{C}_{\alpha_{f}}^{(4)}$ contains $\lambda_{\beta_{h}}$, it is useful to define the following linear combination of constraints:

$$
\tilde{\mathcal{C}}_{\alpha_{f}}^{(3)} \equiv \mathcal{C}_{\alpha_{f}}^{(3)}+\frac{2 k l_{3}}{\kappa_{h 1}} \mathcal{C}_{\beta_{h}}^{(2)}+k \mathcal{C}_{\beta_{f}}^{(2)} \approx 0
$$

$$
\tilde{\mathcal{C}}_{\alpha_{f}}^{(4)} \equiv\left\{\tilde{\mathcal{C}}_{\alpha_{f}}^{(3)}, \mathcal{H}_{T}^{S}\right\} \approx 0
$$

When $\left\{\tilde{\mathcal{C}}_{\alpha_{f}}^{(4)}, \mathcal{C}_{\alpha_{f}}^{(1)}\right\} \neq 0$, the Lagrange multiplier $\lambda_{\alpha_{f}}$ is determined by the time evolution of $\tilde{\mathcal{C}}_{\alpha_{f}}^{(4)}$. In this case, the constraints, $\mathcal{C}_{\beta_{f}}^{(1)}$ and $\tilde{\mathcal{C}}_{\beta_{f}}^{(2,3)}$, still commute with all constraints, and hence, these are first class. The rest of the constraints are second class; therefore, the number of the physical DOFs is given by $(8 \times 2-6-3 \times 2) / 2=2$.

To obtain a theory with $1 \mathrm{DOF}$ in the scalar sector, one more DOF has to be eliminated. Then, we would like to consider the following case:

$$
\begin{aligned}
\left\{\tilde{\mathcal{C}}_{\alpha_{f}}^{(4)}, \mathcal{C}_{\alpha_{f}}^{(1)}\right\} & =\frac{4}{\kappa_{h 1}^{2}}\left(-\frac{3 l_{3}^{2} \mu_{h 1}^{2}}{\kappa_{h 1}}+\frac{\kappa_{f 1}\left(2 \kappa_{h 1} n_{2}+3 l_{3} \mu_{h 1}\right)^{2}}{8 \kappa_{f 1}\left(\kappa_{h 1}+\kappa_{h 4}\right)+3 l_{3}^{2}}\right) \\
& =0 .
\end{aligned}
$$

Solving the above equation, we obtain

$$
\kappa_{h 4}=-\kappa_{h 1}-\frac{3 l_{3}^{2}}{8 \kappa_{f 1}}+\frac{\kappa_{h 1}\left(2 \kappa_{h 1} n_{2}+3 l_{3} \mu_{h 1}\right)^{2}}{24 l_{3}^{2} \mu_{h 1}^{2}} .
$$

In this case, we have two additional constraints:

$\tilde{\mathcal{C}}_{\alpha_{f}}^{(5)} \equiv\left\{\tilde{\mathcal{C}}_{\alpha_{f}}^{(4)}, \mathcal{H}_{T}^{S}\right\} \approx 0, \quad \tilde{\mathcal{C}}_{\alpha_{f}}^{(6)} \equiv\left\{\tilde{\mathcal{C}}_{\alpha_{f}}^{(5)}, \mathcal{H}_{T}^{S}\right\} \approx 0$.

Again, $\tilde{\mathcal{C}}_{\alpha_{f}}^{(6)}$ contains the Lagrange multiplier $\lambda_{\beta_{h}}$, and we redefine the constraint as

$\overline{\mathcal{C}}_{\alpha_{f}}^{(5)} \equiv \tilde{\mathcal{C}}_{\alpha_{f}}^{(5)}+x \mathcal{C}_{\beta_{h}}^{(2)} \approx 0, \quad \overline{\mathcal{C}}_{\alpha_{f}}^{(6)} \equiv\left\{\tilde{\mathcal{C}}_{\alpha_{f}}^{(5)}, \mathcal{H}_{T}^{S}\right\} \approx 0$,

where

$$
x=\frac{k l_{3}}{\kappa_{h 1}^{3}}\left[-2 k^{2} \kappa_{h 1}^{2}+\frac{\mu_{h 1}\left(8 n_{2} \kappa_{f 1} \kappa_{h 1}^{2}-9 l_{3}^{3} \mu_{h 1}\right)}{\kappa_{f 1}\left(2 \kappa_{h 1} n_{2}+3 l_{3} \mu_{h 1}\right)}\right] .
$$

If $\left\{\overline{\mathcal{C}}_{\alpha_{f}}^{(6)}, \mathcal{C}_{\alpha_{f}}^{(1)}\right\} \neq 0$, the Lagrange multiplier $\lambda_{\alpha_{f}}$ can be determined by $\dot{\overline{\mathcal{C}}}_{\alpha_{f}}^{(6)}=\left\{\overline{\mathcal{C}}_{\alpha_{f}}^{(6)}, \mathcal{H}_{T}^{S}\right\} \approx 0$ and no further constraint is generated,

$$
\text { first class: } \mathcal{C}_{\beta_{f}}^{(1)}, \quad \tilde{\mathcal{C}}_{\beta_{f}}^{(2)}, \quad \tilde{\mathcal{C}}_{\beta_{f}}^{(3)},
$$

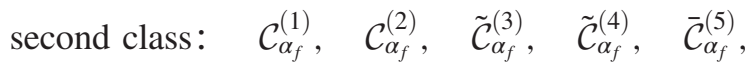

$$
\overline{\mathcal{C}}_{\alpha_{f}}^{(6)}, \quad \mathcal{C}_{\beta_{h}}^{(1)}, \quad \mathcal{C}_{\beta_{h}}^{(2)} .
$$

We finally have

$$
\begin{aligned}
\text { Scalar DOF }= & \frac{1}{2} \times[8 \times 2-8(2 \text { primary \& } 2 \text { secondary \& } 1 \text { tertiary \& } 1 \text { quaternary }+2 \text { more }) \\
& -3(1 \text { primary \& } 1 \text { secondary \& } 1 \text { tertiary }) \times 2 \text { (first-class })]=1 .
\end{aligned}
$$


To summarize, we find a novel class of theory,

(Class Ia):

$$
\begin{aligned}
\kappa_{h 2} & =-\kappa_{h 3}=-2 \kappa_{h 1} \neq 0, \quad \kappa_{f 2}=-\kappa_{f 3}=-2 \kappa_{f 1} \neq 0, \\
\kappa_{f 4} & =-\kappa_{f 1}-\frac{3 l_{3}^{2}}{8 \kappa_{h 1}}, \quad \mu_{f 2}=-\frac{3 l_{3}^{2} \mu_{h 1}}{4 \kappa_{h 1}^{2}} \\
l_{1} & =l_{2}=l_{4}=\mu_{f 1}=n_{1}=0, \quad l_{5}=-l_{3} \\
\kappa_{h 4} & =-\kappa_{h 1}-\frac{3 l_{3}^{2}}{8 \kappa_{f 1}}+\frac{\kappa_{h 1}\left(2 \kappa_{h 1} n_{2}+3 l_{3} \mu_{h 1}\right)^{2}}{24 l_{3}^{2} \mu_{h 1}^{2}}
\end{aligned}
$$

The Lagrangian for class with 1 DOF in the scalar sector Ia is given by

$$
\begin{aligned}
\mathcal{L}= & -\left(\kappa_{h 1} h_{\mu \nu} \hat{\mathcal{E}}^{\mu \nu \alpha \beta} h_{\alpha \beta}+\kappa_{f 1} f_{\mu \nu} \hat{\mathcal{E}}^{\mu \nu \alpha \beta} f_{\alpha \beta}\right)+\delta \kappa_{h 4} h_{, \mu} f^{\mu} \\
& +\frac{3 l_{3}^{2}}{8 \kappa_{h 1}} f_{, \mu} f^{, \mu}+l_{3}\left(h_{, \nu} f_{, \mu}^{\mu \nu}-h_{, \mu}^{\mu \nu} f_{, \nu}\right) \\
& -\mu_{h 1} h_{\mu \nu} h^{\mu \nu}-\mu_{h 2} h^{2}+\left(\frac{3 l_{3}^{2} \mu_{h 1}}{4 \kappa_{h 1}^{2}} f-n_{2} h\right) f,
\end{aligned}
$$

where

$$
\delta \kappa_{h 4}=\frac{3 l_{3}^{2}}{8 \kappa_{f 1}}-\frac{\kappa_{h 1}\left(2 \kappa_{h 1} n_{2}+3 l_{3} \mu_{h 1}\right)^{2}}{24 l_{3}^{2} \mu_{h 1}^{2}} .
$$

One can check that this theory is invariant under the gauge transformation

$$
h_{\mu \nu} \rightarrow \tilde{h}_{\mu \nu}=h_{\mu \nu},
$$

$f_{\mu \nu} \rightarrow \tilde{f}_{\mu \nu}=f_{\mu \nu}+\partial_{\mu} \xi_{\nu}+\partial_{\nu} \xi_{\mu}$ with $\quad \partial^{\mu} \xi_{\mu}=0$.

As one can see from the transverse condition in the gauge transformation, this class is totally distinct from the linearized Hassan-Rosen bigravity, and there are nontrivial kinetic terms for $h$, derivative and mass interactions.

Let us finally discuss the final option where the time evolution of the tertiary constraint $\dot{\tilde{\mathcal{C}}}_{\alpha_{f}}^{(3)}$ does not yield a new constraint. Such a case can be found by rewriting $\dot{\tilde{\mathcal{C}}}_{\alpha_{f}}^{(3)}$ in terms of other constraints, $\mathcal{C}_{\beta_{f}}^{(1)}, \tilde{\mathcal{C}}_{\beta_{f}}^{(2)}, \tilde{\mathcal{C}}_{\beta_{f}}^{(3)}, \mathcal{C}_{\alpha_{f}}^{(1)}, \mathcal{C}_{\alpha_{f}}^{(2)}, \mathcal{C}_{\beta_{h}}^{(1)}$, and $\mathcal{C}_{\beta_{h}}^{(2)}$, and setting it to be zero. Then, we obtain two conditions: Eq. (73) and

$$
\frac{24 l_{3}^{2} \mu_{h 1}^{3}\left(4 n_{2}^{2} \kappa_{h 1}^{2}+3 l_{3}^{2} \mu_{h 1}\left(\mu_{h 1}+4 \mu_{h 2}\right)\right)}{\kappa_{h 1}^{4}\left(2 n_{2} \kappa_{h 1}+3 l_{3} \mu_{h 1}\right)^{2}}=0,
$$

which can be solved for $\mu_{h 2}$,

$$
\mu_{h 2}=-\frac{n_{2}^{2} \kappa_{h 1}^{2}}{3 l_{3}^{2} \mu_{h 1}}-\frac{\mu_{h 1}}{4},
$$

since we assumed $l_{3} \neq 0$ and $\mu_{h 1} \neq 0$. Note that the case where $\left\{\overline{\mathcal{C}}_{\alpha_{f}}^{(6)}, \mathcal{C}_{\alpha_{f}}^{(1)}\right\}$ vanishes in Class Ia reduces to this option. In this case, as shown in (E16), the time evolution of both the tertiary constraints $\tilde{\mathcal{C}}_{\alpha_{f}}^{(3)}$ and $\tilde{\mathcal{C}}_{\beta_{f}}^{(3)}$ can be written in terms of the linear combination of the primary and second class constraints, implying no further constraints. Redefining $\mathcal{C}_{\alpha_{f}}^{(2)}$ as

$$
\tilde{\mathcal{C}}_{\alpha_{f}}^{(2)} \equiv \mathcal{C}_{\alpha_{f}}^{(2)}+\frac{2 k l_{3}}{\kappa_{h 1}} \mathcal{C}_{\beta_{h}}^{(1)}
$$

we find

first class: $\mathcal{C}_{\alpha_{f}}^{(1)}, \quad \tilde{\mathcal{C}}_{\alpha_{f}}^{(2)}, \quad \tilde{\mathcal{C}}_{\alpha_{f}}^{(3)}, \quad \mathcal{C}_{\beta_{f}}^{(1)}, \quad \tilde{\mathcal{C}}_{\beta_{f}}^{(2)}, \quad \tilde{\mathcal{C}}_{\beta_{f}}^{(3)}$,

$$
\text { second class: } \mathcal{C}_{\beta_{h}}^{(1)}, \quad \mathcal{C}_{\beta_{h}}^{(2)} \text {, }
$$

and

Scalar DOF $=\frac{1}{2} \times[8 \times 2-2(1$ primary \& 1 secondary $)$

$$
-6(2 \text { primary \& } 2 \text { secondary \& } 2 \text { tertiary })
$$$$
\times 2 \text { (first-class) }]=1 \text {. }
$$

In this case, the number of the physical DOF is the same as Class Ia, and the resultant theory is invariant under the gauge transformation (84). For this reason, this case can be considered as the special case of Class I by choosing (86) although an additional gauge symmetry is present. To summarize,

(Class Ib):

$$
\begin{aligned}
& \kappa_{h 2}=-\kappa_{h 3}=-2 \kappa_{h 1} \neq 0, \quad \kappa_{f 2}=-\kappa_{f 3}=-2 \kappa_{f 1} \neq 0, \quad \kappa_{f 4}=-\kappa_{f 1}-\frac{3 l_{3}^{2}}{8 \kappa_{h 1}}, \quad \mu_{f 2}=-\frac{3 l_{3}^{2} \mu_{h 1}}{4 \kappa_{h 1}^{2}}, \\
& l_{1}=l_{2}=l_{4}=\mu_{f 1}=n_{1}=0, \quad l_{5}=-l_{3}, \quad \kappa_{h 4}=-\kappa_{h 1}-\frac{3 l_{3}^{2}}{8 \kappa_{f 1}}+\frac{\kappa_{h 1}\left(2 \kappa_{h 1} n_{2}+3 l_{3} \mu_{h 1}\right)^{2}}{24 l_{3}^{2} \mu_{h 1}^{2}} \\
& \mu_{2}=-\frac{n_{2}^{2} \kappa_{h 1}^{2}}{3 l_{3}^{2} \mu_{h 1}}-\frac{\mu_{h 1}}{4} .
\end{aligned}
$$


The Lagrangian for Class $\mathrm{Ib}$ is given by

$$
\begin{aligned}
\mathcal{L}= & -\left(\kappa_{h 1} h_{\mu \nu} \hat{\mathcal{E}}^{\mu \nu \alpha \beta} h_{\alpha \beta}+\kappa_{f 1} f_{\mu \nu} \hat{\mathcal{E}}^{\mu \nu \alpha \beta} f_{\alpha \beta}\right)+\delta \kappa_{h 4} h_{, \mu} f^{, \mu} \\
& +\frac{3 l_{3}^{2}}{8 \kappa_{h 1}} f_{, \mu} f^{\mu}+l_{3}\left(h_{, \nu} f_{, \mu}^{\mu \nu}-h_{, \mu}^{\mu \nu} f_{, \nu}\right)-\mu_{h 1}\left(h_{\mu \nu} h^{\mu \nu}+\frac{1}{4} h^{2}\right)+\frac{1}{3 \mu_{h 1}}\left(\frac{\kappa_{h 1} n_{2}}{l_{3}} h-\frac{3 l_{3} \mu_{h 1}}{2 \kappa_{h 1}} f\right)^{2} .
\end{aligned}
$$

\section{Four primary constraints}

Next, let us consider the case with four primary constraints. As you can see from (56), there are kinetic interactions between $h$ and $f$ fields, which will make the Hamiltonian analysis involved in general. It is interesting to note that we can always map this theory into a simpler theory with two Einstein-Hilbert terms without kinetic interactions between them as explicitly shown in Appendix A 3. Hereinafter we will perform the Hamiltonian analysis in this simple model:

$$
\begin{aligned}
\kappa_{h 2} & =-\kappa_{h 3}=2 \kappa_{h 4}=-2 \kappa_{h 1} \neq 0, \\
\kappa_{f 2} & =-\kappa_{f 3}=2 \kappa_{f 4}=-2 \kappa_{f 1} \neq 0, \\
l_{1} & =l_{2}=l_{3}=l_{4}=l_{5}=0, \quad \mu_{f 1}=n_{1}=0 .
\end{aligned}
$$

Now we have the following four primary constraints:

$$
\begin{array}{ll}
\mathcal{C}_{\alpha_{h}}^{(1)} \equiv \pi_{\alpha_{h}} \approx 0, & \mathcal{C}_{\alpha_{f}}^{(1)} \equiv \pi_{\alpha_{f}} \approx 0, \\
\mathcal{C}_{\beta_{h}}^{(1)} \equiv \pi_{\beta_{h}} \approx 0, & \mathcal{C}_{\beta_{f}}^{(1)} \equiv \pi_{\beta_{f}} \approx 0 .
\end{array}
$$

The total Hamiltonian can be expressed as

$\mathcal{H}_{T}^{S}=\mathcal{H}^{S}+\lambda_{\alpha_{h}} \mathcal{C}_{\alpha_{h}}^{(1)}+\lambda_{\alpha_{f}} \mathcal{C}_{\alpha_{f}}^{(1)}+\lambda_{\beta_{h}} \mathcal{C}_{\beta_{h}}^{(1)}+\lambda_{\beta_{f}} \mathcal{C}_{\beta_{f}}^{(1)}$

The evolution of the primary constraints is given by

$$
\begin{aligned}
\mathcal{C}_{\alpha_{h}}^{(2)} \equiv & \left\{\mathcal{C}_{\alpha_{h}}^{(1)}, \mathcal{H}_{T}^{S}\right\}=-8\left(\mu_{h 1}+\mu_{h 2}\right) \alpha_{h}+8\left(2 k^{2} \kappa_{h 1}\right. \\
& \left.-3 \mu_{h 2}\right) \mathcal{R}_{h}+8 \mu_{h 2} \mathcal{E}_{h}-4 n_{2}\left(\alpha_{f}+3 \mathcal{R}_{f}-\mathcal{E}_{f}\right) \approx 0,
\end{aligned}
$$

$$
\begin{gathered}
\mathcal{C}_{\alpha_{f}}^{(2)} \equiv\left\{\mathcal{C}_{\alpha_{f}}^{(1)}, \mathcal{H}_{T}^{S}\right\}=-8 \mu_{f 2} \alpha_{f}+8\left(2 k^{2} \kappa_{f 1}-3 \mu_{f 2}\right) \mathcal{R}_{f} \\
+8 \mu_{f 2} \mathcal{E}_{f}-4 n_{2}\left(\alpha_{h}+3 \mathcal{R}_{h}-\mathcal{E}_{h}\right) \approx 0, \\
\mathcal{C}_{\beta_{h}}^{(2)} \equiv\left\{\mathcal{C}_{\beta}^{(1)}, \mathcal{H}_{T}^{S}\right\}=-k \pi_{\mathcal{E}_{h}}+4 \mu_{h 1} \beta_{h} \approx 0, \\
0 \approx \mathcal{C}_{\beta_{f}}^{(2)} \equiv\left\{\mathcal{C}_{\beta_{f}}^{(1)}, \mathcal{H}_{T}^{S}\right\}=-k \pi_{\mathcal{E}_{f}} \approx 0 .
\end{gathered}
$$

Here, all the secondary constraints cannot be trivially zero with any choice of the coefficients since $\kappa_{h 1} \neq 0$ and $\kappa_{f 1} \neq 0$. First, let us take a look at the time evolution of the other primary constraints, that is,

$$
\begin{aligned}
\left(\begin{array}{c}
\dot{\mathcal{C}}_{\alpha_{h}}^{(2)} \\
\dot{\mathcal{C}}_{\alpha_{f}}^{(2)} \\
\dot{\mathcal{C}}_{\beta_{h}}^{(2)}
\end{array}\right) & =\left(\begin{array}{c}
\left\{\mathcal{C}_{\alpha_{h}}^{(2)}, \mathcal{H}_{T}^{S}\right\} \\
\left\{\mathcal{C}_{\alpha_{f}}^{(2)}, \mathcal{H}_{T}^{S}\right\} \\
\left\{\mathcal{C}_{\beta_{h}}^{(2)}, \mathcal{H}_{T}^{S}\right\}
\end{array}\right)=\left(\begin{array}{c}
\left\{\mathcal{C}_{\alpha_{h}}^{(2)}, \mathcal{H}^{S}\right\} \\
\left\{\mathcal{C}_{\alpha_{f}}^{(2)}, \mathcal{H}^{S}\right\} \\
\left\{\mathcal{C}_{\beta_{h}}^{(2)}, \mathcal{H}^{S}\right\}
\end{array}\right) \\
& +\left(\begin{array}{ccc}
-8\left(\mu_{h 1}+\mu_{h 2}\right) & -4 n_{2} & 0 \\
-4 n_{2} & -8 \mu_{f 2} & 0 \\
0 & 0 & 4 \mu_{h 1}
\end{array}\right)\left(\begin{array}{c}
\lambda_{\alpha_{h}} \\
\lambda_{\alpha_{f}} \\
\lambda_{\beta_{h}}
\end{array}\right) \approx 0
\end{aligned}
$$

When $n_{2}^{2}-4 \mu_{f 2}\left(\mu_{h 1}+\mu_{h 2}\right) \neq 0$, all the Lagrange multipliers, $\lambda_{\alpha_{h}}, \lambda_{\alpha_{f}}$, and $\lambda_{\beta_{h}}$, are determined by the above equations. As for $\mathcal{C}_{\beta_{f}}^{(2)}$, it commutes with all the primary constraints and the consistency of $\mathcal{C}_{\beta_{f}}^{(2)}$ gives the tertiary constraint

$$
\begin{aligned}
\mathcal{C}_{\beta_{f}}^{(3)} & =\left\{\mathcal{C}_{\beta_{f}}^{(2)}, \mathcal{H}_{T}^{S}\right\} \\
& =-8 k \mu_{f 2}\left(\alpha_{f}+3 \mathcal{R}_{f}-\mathcal{E}_{f}\right)-4 k n_{2}\left(\alpha_{h}+3 \mathcal{R}_{h}-\mathcal{E}_{h}\right) \\
& \approx 0 .
\end{aligned}
$$

Now we redefine the secondary and tertiary constraints for $\beta_{f}$ as

$$
\begin{gathered}
\tilde{\mathcal{C}}_{\beta_{f}}^{(2)}=\mathcal{C}_{\beta_{f}}^{(2)}-k \mathcal{C}_{\alpha_{f}}^{(1)}=-k\left(\pi_{\alpha_{f}}+\pi_{\mathcal{E}_{f}}\right) \approx 0, \\
\tilde{\mathcal{C}}_{\beta_{f}}^{(3)}=\mathcal{C}_{\beta_{f}}^{(3)}-k \mathcal{C}_{\alpha_{f}}^{(2)}=-16 k^{3} \kappa_{f 1} \mathcal{R}_{f} \approx 0 .
\end{gathered}
$$

Then, $\tilde{\mathcal{C}}_{\beta_{f}}^{(3)}$ does commute with $\mathcal{C}_{\alpha_{h}}^{(1)}$ and $\mathcal{C}_{\alpha_{f}}^{(1)}$, and one can see $\dot{\tilde{\mathcal{C}}}_{\beta_{f}}^{(3)}=k^{2} \mathcal{C}_{\beta_{f}}^{(2)} \approx 0$, implying no more constraint is generated. In addition, one can also check that the constraints, $\mathcal{C}_{\beta_{f}}^{(1)}$ and $\tilde{\mathcal{C}}_{\beta_{f}}^{(2,3)}$, commute with all the constraints, and hence these are first class while the rest of the constraints are second class. Therefore, we conclude the number of the physical DOFs is $(8 \times 2-6-3 \times 2) / 2=2$ when $n_{2}^{2}-4 \mu_{f 2}\left(\mu_{h 1}+\mu_{h 2}\right)$ $\neq 0$.

In order to remove an extra DOF, we need to impose an additional constraint for the parameter 


$$
n_{2}^{2}-4 \mu_{f 2}\left(\mu_{h 1}+\mu_{h 2}\right)=0,
$$

which yields two branches,

$$
\begin{gathered}
\mu_{h 2}=-\mu_{h 1}+\frac{n_{2}^{2}}{4 \mu_{f 2}} \quad \text { (Class II), } \\
\mu_{f 2}=n_{2}=0 \quad \text { (Class III). }
\end{gathered}
$$

Note that $\mathcal{C}_{\beta_{f}}^{(3)}$ trivially vanishes in the second case (Class III).

\section{Class II}

Let us consider Class II first. For convenience, we redefine the primary constraint for $\alpha_{h}$ with a linear combination of those for $\alpha_{h}$ and $\alpha_{f}$. Then the four primary constraints read

$$
\begin{aligned}
& \mathcal{C}_{\alpha_{h}}^{(1)} \equiv \pi_{\alpha_{h}}-\frac{n_{2}}{2 \mu_{f 2}} \pi_{\alpha_{f}} \approx 0, \quad \mathcal{C}_{\alpha_{f}}^{(1)} \equiv \pi_{\alpha_{f}} \approx 0, \\
& \mathcal{C}_{\beta_{h}}^{(1)} \equiv \pi_{\beta_{h}} \approx 0, \quad \mathcal{C}_{\beta_{f}}^{(1)} \equiv \pi_{\beta_{f}} \approx 0 .
\end{aligned}
$$

We have the same constraints from the evolution of the primary constraints for $\alpha_{f}, \beta_{h}$, and $\beta_{f}$ as in (97), (98), and (99), respectively. Because of condition (104), only one of the Lagrange multipliers, $\lambda_{\alpha_{h}}$ or $\lambda_{\alpha_{f}}$, is determined by the evolution of $\mathcal{C}_{\alpha_{h}}^{(2)}$ or $\mathcal{C}_{\alpha_{f}}^{(2)}$. Suppose that $\lambda_{\alpha_{f}}$ has been determined by the evolution of $\mathcal{C}_{\alpha_{f}}^{(2)}$ though $\lambda_{\alpha_{h}}$ has not. The evolution of the primary constraint for $\alpha_{h}$ demands

$$
\begin{aligned}
\mathcal{C}_{\alpha_{h}}^{(2)} \equiv\left\{\mathcal{C}_{\alpha_{h}}^{(1)}, \mathcal{H}_{T}^{S}\right\}= & -8 \mu_{h 1} \mathcal{E}_{h}-\frac{8 k^{2} n_{2} \kappa_{f 1}}{\mu_{f 2}} \mathcal{R}_{f} \\
& +8\left(2 k^{2} \kappa_{h 1}+3 \mu_{h 1}\right) \mathcal{R}_{h} \approx 0, \\
\mathcal{C}_{\alpha_{h}}^{(3)} \equiv\left\{\mathcal{C}_{\alpha_{h}}^{(2)}, \mathcal{H}_{T}^{S}\right\}= & -8 k \mu_{h 1} \beta_{h}-\frac{k^{2} n_{2}}{2 \mu_{f 2}} \pi_{\mathcal{E}_{f}}+k^{2} \pi_{\mathcal{E}_{h}} \\
& -\frac{\mu_{h 1}}{2 \kappa_{h 1}} \pi_{\mathcal{R}_{h}} \approx 0 .
\end{aligned}
$$

Since $\mathcal{C}_{\alpha_{h}}^{(3)}$ does not commute with $\mathcal{C}_{\beta_{h}}^{(1)}$, it is convenient to introduce a linear combination of $\mathcal{C}_{\alpha_{h}}^{(3)}$ and $\mathcal{C}_{\beta_{h}}^{(2)}$ as

$$
\tilde{\mathcal{C}}_{\alpha_{h}}^{(3)}=\mathcal{C}_{\alpha_{h}}^{(3)}+2 k \mathcal{C}_{\beta_{h}}^{(2)} .
$$

The evolution of $\tilde{\mathcal{C}}_{\alpha_{h}}^{(3)}$ yields the constraint $\tilde{\mathcal{C}}_{\alpha_{h}}^{(4)}=\left\{\tilde{\mathcal{C}}_{\alpha_{h}}^{(3)}, \mathcal{H}_{T}^{S}\right\}$ $\approx 0$. Since $\left\{\tilde{\mathcal{C}}_{\alpha_{h}}^{(4)}, \mathcal{C}_{\alpha_{h}}^{(1)}\right\}=-12 \mu_{h 1}^{2} / \kappa_{h 1} \neq 0$, the evolution of $\tilde{\mathcal{C}}_{\alpha_{f}}^{(4)}$ determines the Lagrange multiplier $\lambda_{\alpha_{h}}$ and no more constraint is generated. It can easily be checked that $\tilde{\mathcal{C}}_{\alpha_{h}}^{(3)}$ and $\tilde{\mathcal{C}}_{\alpha_{h}}^{(4)}$ cannot be trivially zero. Since in this case

$$
\text { first class: } \mathcal{C}_{\beta_{f}}^{(1)}, \quad \tilde{\mathcal{C}}_{\beta_{f}}^{(2)}, \quad \tilde{\mathcal{C}}_{\beta_{f}}^{(3)},
$$

second class: $\mathcal{C}_{\alpha_{h}}^{(1)}, \quad \mathcal{C}_{\alpha_{h}}^{(2)}, \quad \tilde{\mathcal{C}}_{\alpha_{h}}^{(3)}, \quad \tilde{\mathcal{C}}_{\alpha_{f}}^{(4)}, \quad \mathcal{C}_{\alpha_{f}}^{(1)}$,

$$
\mathcal{C}_{\alpha_{f}}^{(2)}, \quad \mathcal{C}_{\beta_{h}}^{(1)}, \quad \mathcal{C}_{\beta_{h}}^{(2)},
$$

therefore we have

$$
\begin{aligned}
\text { Scalar DOF }= & \frac{1}{2}[8 \times 2-8(3 \text { primary \& } 3 \text { secondary \& } 1 \text { tertiary \& } 1 \text { quaternary }) \\
& -3(1 \text { primary \& } 1 \text { secondary \& } 1 \text { tertiary }) \times 2 \text { (first-class })]=1 .
\end{aligned}
$$

To summarize, we find another novel class of theory with a single DOF in the scalar sector.

Class II:

$$
\begin{aligned}
\kappa_{h 2} & =-\kappa_{h 3}=2 \kappa_{h 4}=-2 \kappa_{h 1} \neq 0, \\
\kappa_{f 2} & =-\kappa_{f 3}=2 \kappa_{f 4}=-2 \kappa_{f 1} \neq 0, \\
l_{1} & =l_{2}=l_{3}=l_{4}=l_{5}=0, \quad \mu_{f 1}=0, \\
n_{1} & =0, \quad \mu_{h 1}+\mu_{h 2}-\frac{n_{2}^{2}}{4 \mu_{f 2}}=0 .
\end{aligned}
$$

The Lagrangian for Class II is given by

$$
\begin{aligned}
\mathcal{L}= & -\left(\kappa_{h 1} h_{\mu \nu} \hat{\mathcal{E}}^{\mu \nu \alpha \beta} h_{\alpha \beta}+\kappa_{f 1} f_{\mu \nu} \hat{\mathcal{E}}^{\mu \nu \alpha \beta} f_{\alpha \beta}\right) \\
& -\mu_{h 1}\left(h_{\mu \nu} h^{\mu \nu}-h^{2}\right)-\frac{1}{4 \mu_{f 2}}\left(n_{2} h+2 \mu_{f 2} f\right)^{2} .
\end{aligned}
$$

One can check that this theory is invariant under the gauge transformation

$$
\begin{gathered}
h_{\mu \nu} \rightarrow \tilde{h}_{\mu \nu}=h_{\mu \nu} \\
f_{\mu \nu} \rightarrow \tilde{f}_{\mu \nu}=f_{\mu \nu}+\partial_{\mu} \xi_{\nu}+\partial_{\nu} \xi_{\mu} \quad \text { with } \quad \partial^{\mu} \xi_{\mu}=0 .
\end{gathered}
$$

Again, because of the transverse condition in the gauge transformation, this theory is different from the linearized Hassan-Rosen bigravity.

\section{Class III}

In this case, we have the same primary constraints as well as the same Hamiltonian as before with the only exception that $\mu_{f 2}=n_{2}=0$ and hence the subsequent constraints are the same. To summarize, we have 
(95): $\mathcal{H}_{T}^{S}=\mathcal{H}^{S}+\lambda_{\alpha_{h}} \mathcal{C}_{\alpha_{h}}^{(1)}+\lambda_{\alpha_{f}} \mathcal{C}_{\alpha_{f}}^{(1)}+\lambda_{\beta_{h}} \mathcal{C}_{\beta_{h}}^{(1)}+\lambda_{\beta_{f}} \mathcal{C}_{\beta_{f}}^{(1)}$,

and

$$
\begin{aligned}
& (94): \mathcal{C}_{\alpha_{h}}^{(1)} \equiv \pi_{\alpha_{h}} \approx 0, \quad \mathcal{C}_{\alpha_{f}}^{(1)} \equiv \pi_{\alpha_{f}} \approx 0, \\
& \mathcal{C}_{\beta_{h}}^{(1)} \equiv \pi_{\beta_{h}} \approx 0, \quad \mathcal{C}_{\beta_{f}}^{(1)} \equiv \pi_{\beta_{f}} \approx 0, \\
& \text { (96-99): } \mathcal{C}_{\alpha_{h}}^{(2)} \approx 0, \quad \mathcal{C}_{\alpha_{f}}^{(2)} \approx 0, \\
& \mathcal{C}_{\beta_{h}}^{(2)} \approx 0, \quad \mathcal{C}_{\beta_{f}}^{(2)} \approx 0 .
\end{aligned}
$$

The Lagrange multipliers $\lambda_{\alpha_{h}}$ and $\lambda_{\beta_{h}}$ are determined from the time evolution of $\mathcal{C}_{\alpha_{h}}^{(2)}$ and $\mathcal{C}_{\beta_{h}}^{(2)}$. This is because $\left\{\mathcal{C}_{\alpha_{h}}^{(2)}, \mathcal{C}_{\alpha_{h}}^{(1)}\right\}=-8\left(\mu_{h 1}+\mu_{h 2}\right)$ and $\left\{\mathcal{C}_{\beta_{h}}^{(2)}, \mathcal{C}_{\beta_{h}}^{(1)}\right\}=4 \mu_{h 1} \neq 0$ with the fact that other Poisson brackets with the primary constraints vanish. In addition, the evolution of $\mathcal{C}_{\alpha_{f}}^{(2)}$ and $\mathcal{C}_{\beta_{f}}^{(2)}$ does not yield a new constraint since

$$
\dot{\mathcal{C}}_{\alpha_{f}}^{(2)}=\left\{\mathcal{C}_{\alpha_{f}}^{(2)}, \mathcal{H}_{T}^{S}\right\}=-k \mathcal{C}_{\beta_{f}}^{(2)} \approx 0,
$$

and $\dot{\mathcal{C}}_{\beta_{f}}^{(2)}=\left\{\mathcal{C}_{\beta_{f}}^{(2)}, \mathcal{H}_{T}^{S}\right\} \approx 0$. Therefore, we find 2 DOFs in the scalar sector,

Scalar DOF $=\frac{1}{2}[8 \times 2-4(2$ primary \& 2 secondary $)$

$$
\begin{aligned}
& -4(2 \text { primary \& } 2 \text { secondary }) \\
& \times 2 \text { (first-class })]=2,
\end{aligned}
$$

since

$$
\text { first class: } \mathcal{C}_{\alpha_{f}}^{(1)}, \quad \mathcal{C}_{\alpha_{f}}^{(2)}, \quad \mathcal{C}_{\beta_{f}}^{(1)}, \quad \mathcal{C}_{\beta_{f}}^{(2)},
$$

$$
\text { second class: } \mathcal{C}_{\alpha_{h}}^{(1)}, \quad \mathcal{C}_{\alpha_{h}}^{(2)}, \quad \mathcal{C}_{\beta_{h}}^{(1)}, \quad \mathcal{C}_{\beta_{h}}^{(2)}
$$

Now the only possible option to have a single DOF is to impose

$$
\mu_{h 1}+\mu_{h 2}=0,
$$

so that we obtain the tertiary constraint from $\alpha_{h}$. In this case the tertiary constraint reads

$\mathcal{C}_{\alpha_{h}}^{(3)}=\left\{\mathcal{C}_{\alpha_{h}}^{(2)}, \mathcal{H}_{T}^{S}\right\}=-8 k \mu_{h 1} \beta_{h}+k^{2} \pi_{\mathcal{E}_{h}}-\frac{\mu_{h 1} \pi_{\mathcal{R}_{h}}}{2 \kappa_{h 1}} \approx 0$.

Since $\mathcal{C}_{\alpha_{h}}^{(3)}$ does not commute with $\mathcal{C}_{\beta_{h}}^{(1)}$, let us define

$$
\tilde{\mathcal{C}}_{\alpha_{h}}^{(3)}=\mathcal{C}_{\alpha_{h}}^{(3)}+2 k \mathcal{C}_{\beta_{h}}^{(2)} .
$$

The evolution of this constraint gives the quaternary constraint:

$$
\begin{aligned}
\tilde{\mathcal{C}}_{\alpha_{h}}^{(4)} & =\left\{\tilde{\mathcal{C}}_{\alpha_{h}}^{(3)}, \mathcal{H}_{T}^{S}\right\} \\
& =\frac{4 \mu_{h 1}}{\kappa_{h 1}}\left[-3 \mu_{h 1} \alpha_{h}+2 \mu_{h 1} \mathcal{E}_{h}+2\left(k^{2} \kappa_{h 1}-3 \mu_{h 1}\right) \mathcal{R}_{h}\right] \\
& \approx 0 .
\end{aligned}
$$

The time evolution of $\tilde{\mathcal{C}}_{\alpha_{h}}^{(4)}$ determines the Lagrange multiplier $\lambda_{\alpha_{h}}$. On the other hand, the evolution of the secondary constraints for $\alpha_{f}, \beta_{h}$, and $\beta_{f}$ do not yield a new constraint. The evolution of $\mathcal{C}_{\alpha_{f}}^{(2)}$ and $\mathcal{C}_{\beta_{f}}^{(2)}$ are trivial since $\dot{\mathcal{C}}_{\alpha_{f}}^{(2)}=\left\{\mathcal{C}_{\alpha_{f}}^{(2)}, \mathcal{H}_{T}^{S}\right\}=-k \mathcal{C}_{\beta_{f}}^{(2)} \quad$ and $\quad \dot{\mathcal{C}}_{\beta_{f}}^{(2)}=\left\{\mathcal{C}_{\beta_{f}}^{(2)}, \mathcal{H}_{T}^{S}\right\}=0$. The time evolution of $\mathcal{C}_{\beta_{h}}^{(2)}$ can be used to determine the Lagrange multiplier, $\lambda_{\beta_{h}}$. Since

$$
\text { first class: } \mathcal{C}_{\alpha_{f}}^{(1)}, \quad \mathcal{C}_{\alpha_{f}}^{(2)}, \quad \mathcal{C}_{\beta_{f}}^{(1)}, \quad \mathcal{C}_{\beta_{f}}^{(2)},
$$

second class: $\mathcal{C}_{\alpha_{h}}^{(1)}, \quad \mathcal{C}_{\alpha_{h}}^{(2)}, \quad \tilde{\mathcal{C}}_{\alpha_{h}}^{(3)}, \quad \tilde{\mathcal{C}}_{\alpha_{h}}^{(4)}, \quad \mathcal{C}_{\beta_{h}}^{(1)}, \quad \mathcal{C}_{\beta_{h}}^{(2)}$,

we find

$$
\begin{aligned}
\text { Scalar DOF }= & \frac{1}{2}[8 \times 2-6(2 \text { primary \& } 2 \text { secondary \& } 1 \text { tertiary \& } 1 \text { quaternary }) \\
& -4(2 \text { primary \& } 2 \text { secondary }) \times 2(\text { first-class })]=1 .
\end{aligned}
$$

In this case

Class III:

$$
\begin{gathered}
\kappa_{h 2}=-\kappa_{h 3}=2 \kappa_{h 4}=-2 \kappa_{h 1} \neq 0, \quad \kappa_{f 2}=-\kappa_{f 3}=2 \kappa_{f 4}=-2 \kappa_{f 1} \neq 0, \\
l_{1}=l_{2}=l_{3}=l_{4}=l_{5}=n_{1}=n_{2}=\mu_{f 1}=\mu_{f 2}=0, \quad \mu_{h 2}=-\mu_{h 1} .
\end{gathered}
$$


The Lagrangian for Class III is given by

$$
\begin{aligned}
\mathcal{L}= & -\left(\kappa_{h 1} h_{\mu \nu} \hat{\mathcal{E}}^{\mu \nu \alpha \beta} h_{\alpha \beta}+\kappa_{f 1} f_{\mu \nu} \hat{\mathcal{E}}^{\mu \nu \alpha \beta} f_{\alpha \beta}\right) \\
& -\mu_{h 1}\left(h_{\mu \nu} h^{\mu \nu}-h^{2}\right) .
\end{aligned}
$$

It is clear that this case corresponds to the linearized Hassan-Rosen bigravity, (10).

\section{SUMMARY}

In this paper, we investigated a Lorentz invariant action for two rank-2 symmetric tensor fields $h_{\mu \nu}$ and $f_{\mu \nu}$. Based on the Hamiltonian analysis, we classified theories with 7 physical degrees of freedom whose action consists of the most generic quadratic terms containing up to two derivatives with respect to spacetime for each term. To simplify the problem, we have utilized a field redefinition to reduce the model parameter space. We then found three distinct classes of theories, which are not connected by a linear field redefinition. In any case, the Hamiltonian structure in the tensor and vector sectors are the same; that is, one of the fields behaves as massless, and the other has a nonvanishing mass in dispersion relations. The first theory, Class I, contains three primary constraints in the scalar sector and is invariant under the transverse diffeomorphism. Furthermore, the kinetic terms for both fields do not take the form of the Einstein-Hilbert term even by the field redefinition, and the mass term no longer has the Fierz-Pauli tuning. Class II is also invariant under the transverse diffeomorphism but contains four primary constraints differently from Class I. The kinetic terms for both fields are described by the EinsteinHilbert terms, and a new tuning parameter enters in the mass matrix thanks to the transverse condition in the gauge transformation, which was absent in the linearized Hassan-Rosen bigravity. Class III is nothing but the linearized Hassan-Rosen bigravity, which is invariant under the standard diffeomorphism. Since we have reduced the model parameter space by the linear field redefinition before the Hamiltonian analysis, a broader class of theories can be obtained by the field redefinition, which could be different theories depending on the matter coupling, although their Hamiltonian properties and physical degrees of freedom do not change. It should again be stressed that neither Class II nor Class III can be mapped into Class I through any field redefinition since the number of the primary constraints does not change under the transformation.

The transverse diffeomorphism that appeared in Classes I and II can be nonlinearized by introducing the unimodular condition $\operatorname{det} g=1$, where $g$ is one of the metrics in bimetric gravity. Therefore, the first two classes of theories, Class I and Class II, might open a new window of finding extended theories of massive bimetric gravity. In fact, if we linearize the Hassan-Rosen bigravity with the unimodular condition, one is able to obtain a part of Class II, where all the mixing terms are switched off. Although such a case is trivial because the unimodular condition brings just a cosmological constant in the Einstein equation as the (massless) unimodular gravity, it would be interesting to investigate whether nonlinear completions of Class II itself can be possible or not. Moreover, the nonlinearization of Class I would also be interesting.

\section{ACKNOWLEDGMENTS}

We are grateful to Norihiro Tanahashi for the initial collaboration in the early stage of this work. A. N. also thanks Takahiro Tanaka for fruitful discussion and useful suggestions. This work was supported in part by JSPS Grants-in-Aid for Scientific Research No. JP17K14304 (D. Y.), No. JP19H01891 (A. N. and D. Y.), and No. $20 \mathrm{H} 05852$ (A. N.).

\section{APPENDIX A: LINEAR FIELD REDEFINITION}

In this appendix, we consider the transformation of the action for the fields $h_{\mu \nu}$ and $f_{\mu \nu}$ under a redefinition of them. The most generic transformation linear in the fields ${ }^{3}$ is

$$
\begin{aligned}
& h_{\mu \nu}=\Omega_{h} \bar{h}_{\mu \nu}+\omega_{h} \bar{f}_{\mu \nu}+\left(\Gamma_{h} \bar{h}+\gamma_{h} \bar{f}\right) \eta_{\mu \nu}, \\
& f_{\mu \nu}=\Omega_{f} \bar{f}_{\mu \nu}+\omega_{f} \bar{h}_{\mu \nu}+\left(\Gamma_{f} \bar{f}+\gamma_{f} \bar{h}\right) \eta_{\mu \nu},
\end{aligned}
$$

where $\Omega_{h, f}$ and $\Gamma_{h, f}$ are constants and $\bar{h}$ and $\bar{f}$ are the traces of $\bar{h}_{\mu \nu}$ and $\bar{f}_{\mu \nu}$ contracted by $\eta_{\mu \nu}$. Since $\Omega_{h}$ and $\Omega_{f}$ only change the normalization for each Lagrangian, we hereafter set $\Omega_{h}=\Omega_{f}=1$. Applying the transformation to the generic action, one obtains

$$
\begin{aligned}
S= & \int \mathrm{d}^{4} x\left(-\overline{\mathcal{K}}_{h}^{\alpha \beta \mid \mu \nu \rho \sigma} h_{\mu \nu, \alpha} h_{\rho \sigma, \beta}-\overline{\mathcal{K}}_{f}^{\alpha \beta \mid \mu \nu \rho \sigma} f_{\mu \nu, \alpha} f_{\rho \sigma, \beta}\right. \\
& -\overline{\mathcal{G}}^{\alpha \beta \mu \nu \rho \sigma} h_{\mu \nu, \alpha} f_{\rho \sigma, \beta}-\overline{\mathcal{M}}_{h}^{\mu \nu \rho \sigma} h_{\mu \nu} h_{\rho \sigma} \\
& \left.-\overline{\mathcal{M}}_{f}^{\mu \nu \rho \sigma} f_{\mu \nu} f_{\rho \sigma}-\overline{\mathcal{N}}^{\mu \nu \rho \sigma} h_{\mu \nu} f_{\rho \sigma}\right),
\end{aligned}
$$

where the coefficients of the transformed Lagrangian read

$$
\begin{aligned}
& \bar{\kappa}_{h 1}=\kappa_{h 1}+\omega_{f}\left(l_{1}+\omega_{f} \kappa_{f 1}\right), \\
& \bar{\kappa}_{h 2}=\kappa_{h 2}+\omega_{f}\left(l_{2}+\omega_{f} \kappa_{f 2}\right), \\
\bar{\kappa}_{h 3}= & 2 \Gamma_{h} \kappa_{h 2}+\left(1+4 \Gamma_{h}\right) \kappa_{h 3} \\
& +\omega_{f}\left[2 \gamma_{f} \kappa_{f 2}+\left(\omega_{f}+4 \gamma_{f}\right) \kappa_{f 3}\right] \\
& +\left(\gamma_{f}+\Gamma_{h} \omega_{f}\right) l_{2}+\left(\omega_{f}+4 \gamma_{f}\right) l_{3} \\
& +\omega_{f}\left(1+4 \Gamma_{h}\right) l_{5},
\end{aligned}
$$

\footnotetext{
${ }^{3}$ One can also consider other invertible transformations involving derivatives such as $h_{\mu \nu} \rightarrow h_{\mu \nu}+\partial_{\mu} \partial_{\nu} f$ and $f_{\mu \nu} \rightarrow f_{\mu \nu}$ $+\partial_{\mu} \partial_{\nu} h$. However, these transformations introduces higher derivatives, and the resultant action will be no longer the form of the action (5) even after imposing the conditions (80), (91), (114), and (132).
} 


$$
\begin{aligned}
\bar{\kappa}_{h 4}= & 2 \Gamma_{h}\left(1+2 \Gamma_{h}\right) \kappa_{h 1}+\Gamma_{h}^{2} \kappa_{h 2}+\Gamma_{h}\left(1+4 \Gamma_{h}\right) \kappa_{h 3}+\left(1+4 \Gamma_{h}\right)^{2} \kappa_{h 4} \\
& +2 \gamma_{f}\left(\omega_{f}+2 \gamma_{f}\right) \kappa_{f 1}+\gamma_{f}^{2} \kappa_{f 2}+\gamma_{f}\left(\omega_{f}+4 \Gamma_{f}\right) \kappa_{f 3}+\left(\omega_{f}+4 \gamma_{f}\right)^{2} \kappa_{f 4} \\
& +\left[\gamma_{f}\left(1+4 \Gamma_{h}\right)+\omega_{f} \Gamma_{h}\right] l_{1}+\gamma_{f} \Gamma_{h} l_{2}+\Gamma_{h}\left(\omega_{f}+4 \gamma_{f}\right) l_{3}+\left(1+4 \Gamma_{h}\right)\left(\omega_{f}+4 \gamma_{f}\right) l_{4}+\gamma_{f}\left(1+4 \Gamma_{h}\right) l_{5},
\end{aligned}
$$

$$
\bar{\mu}_{h 1}=\mu_{h 1}+\omega_{f}^{2} \mu_{f 1}+\omega_{f} n_{1},
$$

$$
\begin{gathered}
\bar{l}_{1}=\left(1+\omega_{h} \omega_{f}\right) l_{1}+2 \omega_{h} \kappa_{h 1}+2 \omega_{f} \kappa_{f 1}, \\
\bar{l}_{2}=\left(1+\omega_{h} \omega_{f}\right) l_{2}+2 \omega_{h} \kappa_{h 2}+2 \omega_{f} \kappa_{f 2}, \\
\bar{l}_{3}=\left(\Gamma_{f}+\omega_{f} \gamma_{h}\right) l_{2}+\left(1+4 \Gamma_{f}\right) l_{3}+\omega_{f}\left(\omega_{h}+4 \gamma_{h}\right) l_{5} \\
+2 \gamma_{h} \kappa_{h 2}+\left(\omega_{h}+4 \gamma_{h}\right) \kappa_{h 3}+2 \omega_{f} \Gamma_{f} \kappa_{f 2} \\
+\omega_{f}\left(1+4 \Gamma_{f}\right) \kappa_{f 3},
\end{gathered}
$$$$
+\left[2 \gamma_{f}\left(\omega_{f}+2 \gamma_{f}\right) \mu_{f 1}+\left(\omega_{f}+4 \gamma_{f}\right)^{2} \mu_{f 2}\right]
$$$$
+\left[\Gamma_{h} \omega_{f}+\left(1+4 \Gamma_{f}\right) \gamma_{f}\right] n_{1}+\left(1+4 \Gamma_{h}\right)\left(\omega_{f}+4 \gamma_{f}\right) n_{2},
$$

and $\bar{\kappa}_{f 1, f 2, f 3, f 4}$ and $\bar{\mu}_{f 1, f 2}$ can be obtained by replacing the labels $h$ and $f$. And also we find

$$
\begin{gathered}
\bar{l}_{4}=\left(\Gamma_{h}+\Gamma_{f}+4 \Gamma_{h} \Gamma_{f}+\omega_{h} \gamma_{f}+\omega_{f} \gamma_{h}+4 \gamma_{h} \gamma_{f}\right) l_{1}+\left(\Gamma_{h} \Gamma_{f}+\gamma_{h} \gamma_{f}\right) l_{2}+\left[\Gamma_{h}\left(1+4 \Gamma_{f}\right)+\gamma_{h}\left(\omega_{f}+4 \gamma_{f}\right)\right] l_{3} \\
+\left[\left(1+4 \Gamma_{h}\right)\left(1+4 \Gamma_{f}\right)+\left(\omega_{h}+4 \gamma_{h}\right)\left(\omega_{f}+4 \gamma_{f}\right)\right] l_{4}+\left[\Gamma_{f}\left(1+4 \Gamma_{h}\right)+\gamma_{f}\left(\omega_{h}+4 \gamma_{h}\right)\right] l_{5} \\
+2\left[\gamma_{h}\left(1+4 \Gamma_{h}\right)+\omega_{h} \Gamma_{h}\right] \kappa_{h 1}+2 \gamma_{h} \Gamma_{h} \kappa_{h 2}+\left[\gamma_{h}\left(1+8 \Gamma_{h}\right)+\omega_{h} \Gamma_{h}\right] \kappa_{h 3}+2\left(1+4 \Gamma_{h}\right)\left(\omega_{h}+4 \gamma_{h}\right) \kappa_{h 4} \\
+2\left[\gamma_{f}\left(1+4 \Gamma_{f}\right)+\omega_{f} \Gamma_{f}\right] \kappa_{f 1}+2 \gamma_{f} \Gamma_{f} \kappa_{f 2}+\left[\gamma_{f}\left(1+8 \Gamma_{f}\right)+\omega_{f} \Gamma_{f}\right] \kappa_{f 3}+2\left(1+4 \Gamma_{f}\right)\left(\omega_{f}+4 \gamma_{f}\right) \kappa_{f 4}, \\
\bar{l}_{5}=\left(\Gamma_{h}+\omega_{h} \gamma_{f}\right) l_{2}+\omega_{h}\left(\omega_{f}+4 \gamma_{f}\right) l_{3}+\left(1+4 \Gamma_{h}\right) l_{5}+2 \gamma_{f} \kappa_{f 2}+\left(\omega_{f}+4 \gamma_{f}\right) \kappa_{f 3}+2 \omega_{h} \Gamma_{h} \kappa_{h 2}+\omega_{h}\left(1+4 \Gamma_{h}\right) \kappa_{h 3}, \\
\bar{n}_{1}=\left(1+\omega_{h} \omega_{f}\right) n_{1}+2 \omega_{h} \mu_{h 1}+2 \omega_{f} \mu_{f 1}, \\
\bar{n}_{2}=\left(\Gamma_{h}+\Gamma_{f}+4 \Gamma_{h} \Gamma_{f}+\omega_{h} \gamma_{f}+\omega_{f} \gamma_{h}+4 \gamma_{h} \gamma_{f}\right) n_{1}+\left[\left(1+4 \Gamma_{h}\right)\left(1+4 \Gamma_{f}\right)\right. \\
+\left[\left(\omega_{h}+4 \gamma_{h}\right)\left(\omega_{f}+4 \gamma_{f}\right)\right] n_{2}+2\left[\gamma_{h}\left(1+4 \Gamma_{h}\right)+\omega_{h} \Gamma_{h}\right] \mu_{h 1}+2\left(1+4 \Gamma_{h}\right)\left(\omega_{h}+4 \gamma_{h}\right) \mu_{h 2} \\
+2\left[\gamma_{f}\left(1+4 \Gamma_{f}\right)+\omega_{f} \Gamma_{f}\right] \mu_{f 1}+2\left(1+4 \Gamma_{f}\right)\left(\omega_{f}+4 \gamma_{f}\right) \mu_{f 2} .
\end{gathered}
$$

The inverse transformation of the fields is given by

$$
\begin{aligned}
& \bar{f}_{\mu \nu}=\frac{1}{4}\left\{\frac{4 h_{\mu \nu}-4 \omega_{h} f_{\mu \nu}-\left(h-\omega_{h} f\right) \eta_{\mu \nu}}{1-\omega_{h} \omega_{f}}+\frac{\left(1+4 \Gamma_{f}\right) h-\left(\omega_{h}+4 \gamma_{h}\right) f}{\left(1+4 \Gamma_{h}\right)\left(1+4 \Gamma_{f}\right)-\left(\omega_{f}+4 \gamma_{f}\right)\left(\omega_{h}+4 \gamma_{h}\right)} \eta_{\mu \nu}\right\}, \\
& \bar{f}_{\mu \nu}=\frac{1}{4}\left\{\frac{4 f_{\mu \nu}-4 \omega_{f} h_{\mu \nu}-\left(f-\omega_{f} h\right) \eta_{\mu \nu}}{1-\omega_{h} \omega_{f}}+\frac{\left(1+4 \Gamma_{h}\right) f-\left(\omega_{f}+4 \gamma_{f}\right) h}{\left(1+4 \Gamma_{h}\right)\left(1+4 \Gamma_{f}\right)-\left(\omega_{f}+4 \gamma_{f}\right)\left(\omega_{h}+4 \gamma_{h}\right)} \eta_{\mu \nu}\right\},
\end{aligned}
$$

and that for the trace of the fields

$$
\begin{aligned}
& \bar{h}=\frac{\left(1+4 \Gamma_{f}\right) h-\left(\omega_{h}+4 \gamma_{h}\right) f}{1+4 \Gamma_{h}+4 \Gamma_{f}\left(1+4 \Gamma_{h}\right)-\left(\omega_{f}+4 \gamma_{f}\right)\left(\omega_{h}+4 \gamma_{h}\right)}, \\
& \bar{f}=\frac{\left(1+4 \Gamma_{h}\right) f-\left(\omega_{f}+4 \gamma_{f}\right) h}{1+4 \Gamma_{h}+4 \Gamma_{f}\left(1+4 \Gamma_{h}\right)-\left(\omega_{f}+4 \gamma_{f}\right)\left(\omega_{h}+4 \gamma_{h}\right)},
\end{aligned}
$$

where the inverse transformation exists only when

$$
\begin{gathered}
1-\omega_{h} \omega_{f} \neq 0 \\
1+4 \Gamma_{h}+4 \Gamma_{f}\left(1+4 \Gamma_{h}\right)-\left(\omega_{f}+4 \gamma_{f}\right)\left(\omega_{h}+4 \gamma_{h}\right) \neq 0 .
\end{gathered}
$$




\section{Transformation under vector conditions}

In this appendix, we show that one can impose (47) by using the field redefinition, without loss of generality. Here, we consider a specific field redefinition under the vector condition (44) to simplify the analysis. Let us first consider the following transformation:

$$
h_{\mu \nu}=\bar{h}_{\mu \nu}-\frac{l_{1}}{2 \kappa_{h 1}} \bar{f}_{\mu \nu}, \quad f_{\mu \nu}=\bar{f}_{\mu \nu}
$$

and then one can find $\bar{l}_{1}=0$ in the transformed theories. Moreover, in the case of $\kappa_{h 3} \neq \kappa_{h 1}$ and $\kappa_{f 3} \neq \kappa_{f 1}$, if one considers the field transformation defined as

$$
\begin{aligned}
& h_{\mu \nu}=\bar{h}_{\mu \nu}-\frac{2 \kappa_{h 1}-\kappa_{h 3}}{2\left(\kappa_{h 1}-\kappa_{h 3}\right)} \bar{h} \eta_{\mu \nu}, \\
& f_{\mu \nu}=\bar{f}_{\mu \nu}-\frac{2 \kappa_{f 1}-\kappa_{f 3}}{2\left(\kappa_{f 1}-\kappa_{f 3}\right)} \bar{f} \eta_{\mu \nu},
\end{aligned}
$$

one can transform to the theories with $\bar{\kappa}_{h 3}=2 \bar{\kappa}_{h 1}$ and $\bar{\kappa}_{f 3}=$ $2 \bar{\kappa}_{f 1}$ with the use of the first two conditions of (44). Next, when one considers the following transformation:

$$
\begin{aligned}
& h_{\mu \nu}=\bar{h}_{\mu \nu}+\left(-\frac{l_{3}}{\kappa_{h 1}} \gamma_{f} \bar{h}+\gamma_{h} \bar{f}\right) \eta_{\mu \nu}, \\
& f_{\mu \nu}=\bar{f}_{\mu \nu}+\left(-\frac{l_{5}}{\kappa_{f 1}} \gamma_{h} \bar{f}+\gamma_{f} \bar{h}\right) \eta_{\mu \nu},
\end{aligned}
$$

one can check that the transformed parameters still satisfy the conditions: $\bar{\kappa}_{h 3}=2 \bar{\kappa}_{h 1}$ and $\bar{\kappa}_{f 3}=2 \bar{\kappa}_{f 1}$. Under these conditions, using the transformation Eqs. (A25) and (A26) in which only $\gamma_{f}$ is considered, one finds

$$
\begin{aligned}
\bar{l}_{3}+\bar{l}_{4}+\bar{l}_{5}= & l_{3}+l_{4}+l_{5} \\
& +\left[8\left(\kappa_{f 1}+\kappa_{f 4}\right)-\frac{l_{3}\left(l_{3}+4 l_{4}+4 l_{5}\right)}{\kappa_{h 1}}\right] \gamma_{f} .
\end{aligned}
$$

Hence, performing the transformation Eqs. (A25) and (A26) with

$\gamma_{f}=-\frac{\kappa_{h 1}\left(l_{3}+l_{4}+l_{5}\right)}{l_{3}\left(l_{3}+4 l_{4}+4 l_{5}\right)-8 \kappa_{h 1}\left(\kappa_{f 1}+\kappa_{f 4}\right)}, \quad \gamma_{h}=0$,

one can transform to the theories with $\bar{\kappa}_{h 3}=2 \bar{\kappa}_{h 1}$, $\bar{\kappa}_{f 3}=2 \bar{\kappa}_{f 1}$, and $\bar{l}_{3}+\bar{l}_{4}+\bar{l}_{5}=0$.

\section{Three primary case in the scalar sector}

In this appendix, we show that the conditions (58) can be imposed by the field redefinition, without loss of generality. Let us first consider the first case of (54) for the original theory described by $h_{\mu \nu}$. In order to simply the Lagrangian, we impose

$$
\begin{aligned}
\bar{l}_{1} & =\bar{l}_{4}=0, \quad \bar{l}_{3}+\bar{l}_{5}=0, \quad \bar{\kappa}_{h 3}=2 \bar{\kappa}_{h 1}, \\
\bar{\kappa}_{f 3} & =2 \bar{\kappa}_{f 1}, \quad \bar{n}_{1}=0 .
\end{aligned}
$$

These conditions determine the coefficients of the field redefinition as follows:

$$
\begin{aligned}
& \omega_{h}=-\frac{n_{1}}{2 \mu_{h 1}}, \quad \omega_{f}=\frac{n_{1} \kappa_{h 1}}{2 \mu_{h 1} \kappa_{f 1}}, \\
& \Gamma_{h}=-\frac{-2 l_{3} \mu_{h 1}\left(l_{3} l_{5} n_{1}-2\left(l_{3}+l_{5}\right) \mu_{h 1} \kappa_{f 1}\right)+l_{3} n_{1}\left(l_{3} n_{1}+2 \mu_{h 1} \kappa_{f 1}\right) \kappa_{h 1}+n_{1}^{2} \kappa_{f 1} \kappa_{h 1}^{2}}{8 \mu_{h 1}\left(-l_{3} n_{1}+2 \mu_{h 1} \kappa_{f 1}\right)\left(l_{3} l_{5}-\kappa_{f 1} \kappa_{h 1}\right)}, \quad \Gamma_{f}=\frac{n_{1}\left(2 l_{3} \mu_{h 1}+n_{1} \kappa_{h 1}\right)}{8 \mu_{h 1}\left(2 \mu_{h 1} \kappa_{f 1}-l_{3} n_{1}\right)}, \\
& \gamma_{h}=\frac{n_{1}}{8 \mu_{h 1}}, \quad \gamma_{f}=-\frac{\kappa_{h 1}}{\kappa_{f 1}} \frac{l_{3}^{2} l_{5} n_{1}^{2}+2 l_{3} \mu_{h 1} \kappa_{f 1}\left(2 \mu_{h 1} \kappa_{f 1}-l_{5} n_{1}\right)+\kappa_{f 1}\left(4 l_{5} \mu_{h 1}^{2} \kappa_{f 1}+n_{1}\left(l_{5} n_{1}+2 \mu_{h 1} \kappa_{f 1}\right) \kappa_{h 1}\right)}{8 \mu_{h 1}\left(l_{3} n_{1}-2 \mu_{h 1} \kappa_{f 1}\right)\left(l_{3} l_{5}-\kappa_{f 1} \kappa_{h 1}\right)} .
\end{aligned}
$$

Then the transformed Lagrangian satisfies

$$
\begin{aligned}
& \kappa_{h 2}=-\kappa_{h 3}=-2 \kappa_{h 1} \neq 0, \quad \kappa_{f 2}=-\kappa_{f 3}=-2 \kappa_{f 1} \neq 0, \\
& \kappa_{f 4}=-\kappa_{f 1}-\frac{3 l_{3}^{2}}{8 \kappa_{h 1}}, \quad l_{1}=l_{2}=l_{4}=\mu_{f 1}=n_{1}=0, \\
& l_{5}=-l_{3} .
\end{aligned}
$$

Here the bars are omitted. Thus the kinetic term for $f_{\mu \nu}$ is the Einstein-Hilbert term, and all kinetic interactions between $h$ and $f$ are absent in this frame. Since these conditions are the same as (58), we conclude that the theories having the first option of (54) are transformed to the theories with (58).

Next let us consider the second case of (54). Imposing the same conditions (A29), we find the transformation, 


$$
\begin{aligned}
& \omega_{h}=-\frac{n_{1}}{2 \mu_{h 1}}, \quad \omega_{f}=\frac{n_{1} \kappa_{h 1}}{2 \mu_{h 1} \kappa_{f 1}}, \\
& \Gamma_{h}=-\frac{4 l_{3} l_{5}^{2} \mu_{h 1}^{2}+2 l_{3} \mu_{h 1}\left(-l_{5} n_{1}+2 \mu_{h 1} \kappa_{f 1}\right) \kappa_{h 1}+n_{1}\left(\left(l_{3}+l_{5}\right) n_{1}+2 \mu_{h 1} \kappa_{f 1}\right) \kappa_{h 1}^{2}}{8 \mu_{h 1}\left(2 l_{5} \mu_{h 1}-n_{1} \kappa_{h 1}\right)\left(l_{3} l_{5}-\kappa_{f 1} \kappa_{h 1}\right)}, \quad \Gamma_{f}=-\frac{1}{4}, \\
& \gamma_{h}=\frac{l_{5} n_{1}+2 \mu_{h 1} \kappa_{f 1}}{8 l_{5} \mu_{h 1}-4 n_{1} \kappa_{h 1}}, \quad \gamma_{f}=-\frac{\kappa_{h 1}}{\kappa_{f 1}} \frac{2 l_{5}^{2} \mu_{h 1}\left(l_{3} n_{1}-2 \mu_{h 1} \kappa_{f 1}\right)-\left(l_{5}\left(l_{3}+l_{5}\right) n_{1}^{2}+2 l_{5} \mu_{h 1} n_{1} \kappa_{f 1}+4 \mu_{h 1}^{2} \kappa_{f 1}^{2}\right) \kappa_{h 1}}{8 \mu_{h 1}\left(2 l_{5} \mu_{h 1}-n_{1} \kappa_{h 1}\right)\left(l_{3} l_{5}-\kappa_{f 1} \kappa_{h 1}\right)},
\end{aligned}
$$

where the transformed Lagrangian satisfies (A31) equivalent to (58). Therefore, both the first and second options of the three primary case (54) can be mapped into (58).

\section{Four primary case in the scalar sector}

In this appendix, we show that conditions (93) can be imposed using the field redefinition without loss of generality. Let us now consider the four primary case (56) for the original theory described by $h_{\mu \nu}$. In order to simplify the Lagrangian, we here impose

$$
\begin{gathered}
\bar{l}_{1}=0, \quad \bar{\kappa}_{h 3}=2 \bar{\kappa}_{h 1}, \quad \bar{\kappa}_{f 3}=2 \bar{\kappa}_{f 1}, \\
\bar{\kappa}_{h 4}=-\bar{\kappa}_{h 1}, \quad \bar{\kappa}_{f 4}=-\bar{\kappa}_{f 1}, \quad \bar{\mu}_{f 1}=0 .
\end{gathered}
$$

These conditions provide the field redefinition with the following coefficients:

$$
\begin{gathered}
\omega_{h}=-\frac{n_{1}}{2 \mu_{h 1}}, \quad \omega_{f}=\frac{n_{1} \kappa_{h 1}}{2 \mu_{h 1} \kappa_{f 1}}, \\
\Gamma_{h}=-\frac{2 l_{3} l_{5} \mu_{h 1}-l_{3} n_{1} \kappa_{h 1}}{8 \mu_{h 1}\left(l_{3} l_{5}-\kappa_{f 1} \kappa_{h 1}\right)} \\
\Gamma_{f}=-\frac{2 l_{3} l_{5} \mu_{h 1}+l_{5} n_{1} \kappa_{h 1}}{8 \mu_{h 1}\left(l_{3} l_{5}-\kappa_{f 1} \kappa_{h 1}\right)} \\
\gamma_{h}=\frac{l_{3} l_{5} n_{1}+2 l_{3} \mu_{h 1} \kappa_{f 1}}{8 \mu_{h 1}\left(l_{3} l_{5}-\kappa_{f 1} \kappa_{h 1}\right)}, \\
\gamma_{f}=-\frac{\kappa_{h 1}\left(l_{3} l_{5} n_{1}-2 l_{5} \kappa_{f 1} \mu_{h 1}\right)}{8 \mu_{h 1} \kappa_{f 1}\left(l_{3} l_{5}-\kappa_{f 1} \kappa_{h 1}\right)} .
\end{gathered}
$$

Then the transformed Lagrangian satisfies

$$
\begin{aligned}
\kappa_{h 2} & =-\kappa_{h 3}=2 \kappa_{h 4}=-2 \kappa_{h 1} \neq 0, \\
\kappa_{f 2} & =-\kappa_{f 3}=2 \kappa_{f 4}=-2 \kappa_{f 1} \neq 0, \\
l_{1} & =l_{2}=l_{3}=l_{4}=l_{5}=0, \quad \mu_{f 1}=n_{1}=0,
\end{aligned}
$$

which is obviously equivalent to (93). Here we omit the bar of the coefficients. Thus in the four primary case, we can always map them into two Einstein-Hilbert terms with no kinetic interactions between $h$ and $f$.

\section{APPENDIX B: LAGRANGIAN IN THE SCALAR SECTOR}

The Lagrangian for $h$ scalar perturbations is given by

$$
\begin{aligned}
\mathcal{L}_{h h, \mathrm{kin}}^{S}= & 4\left(\kappa_{h 1}+\kappa_{h 2}+\kappa_{h 3}+\kappa_{h 4}\right) \dot{\alpha}_{h}^{2}-\left(2 \kappa_{h 1}+\kappa_{h 2}\right) \dot{\beta}_{h}^{2} \\
& +12\left(\kappa_{h 1}+3 \kappa_{h 4}\right) \dot{\mathcal{R}}_{h}^{2}+4\left(\kappa_{h 1}+\kappa_{h 4}\right) \dot{\mathcal{E}}_{h}^{2} \\
& -4\left(\kappa_{h 3}+2 \kappa_{h 4}\right)\left(-3 \dot{\mathcal{R}}_{h}+\dot{\mathcal{E}}_{h}\right) \dot{\alpha}_{h} \\
& -8\left(\kappa_{h 1}+3 \kappa_{h 4}\right) \dot{\mathcal{R}}_{h} \dot{\mathcal{E}}_{h}, \\
\mathcal{L}_{h h, \text { cross }}^{S}= & -4\left[\left(\kappa_{h 2}+\kappa_{h 3}\right) \dot{\alpha}_{h}+\left(\kappa_{h 2}+3 \kappa_{h 3}\right) \dot{\mathcal{R}}_{h}\right. \\
& \left.-\left(\kappa_{h 2}+\kappa_{h 3}\right) \dot{\mathcal{E}}_{h}\right] k \beta_{h},
\end{aligned}
$$

The Lagrangian for $f$ perturbations can be obtained by replacing the above Lagrangian for $h$ with $f$ :

$$
\begin{aligned}
\mathcal{L}_{h f, \text { kin }}^{S}= & 4\left(l_{2}+l_{3}+l_{4}+l_{5}\right) \dot{\alpha}_{h} \dot{\alpha}_{f}-l_{2} \dot{\beta}_{h} \dot{\beta}_{f}+36 l_{4} \dot{\mathcal{R}}_{h} \dot{\mathcal{R}}_{f}+4 l_{4} \dot{\mathcal{E}}_{h} \dot{\mathcal{E}}_{f}+12\left(l_{3}+l_{4}\right) \dot{\alpha}_{h} \dot{\mathcal{R}}_{f}+12\left(l_{4}+l_{5}\right) \dot{\alpha}_{f} \dot{\mathcal{R}}_{h} \\
& -4\left(l_{3}+l_{4}\right) \dot{\alpha}_{h} \dot{\mathcal{E}}_{f}-4\left(l_{4}+l_{5}\right) \dot{\alpha}_{f} \dot{\mathcal{E}}_{h}-12 l_{4}\left(\dot{\mathcal{R}}_{h} \dot{\mathcal{E}}_{f}+\dot{\mathcal{R}}_{f} \dot{\mathcal{E}}_{h}\right),
\end{aligned}
$$




$$
\begin{aligned}
\mathcal{L}_{h f, \text { cross }}^{S}= & -2\left[\left(l_{2}+2 l_{5}\right) \dot{\alpha}_{h}+\left(l_{2}+6 l_{5}\right) \dot{\mathcal{R}}_{h}-\left(l_{2}+2 l_{5}\right) \dot{\mathcal{E}}_{h}\right] k \beta_{f}-2\left[\left(l_{2}+2 l_{3}\right) \dot{\alpha}_{f}+\left(l_{2}+6 l_{3}\right) \dot{\mathcal{R}}_{f}-\left(l_{2}+2 l_{3}\right) \dot{\mathcal{E}}_{f}\right] k \beta_{h}, \\
\mathcal{L}_{h f \text {, mass }}^{S}= & -4\left(k^{2} l_{4}+n_{1}+n_{2}\right) \alpha_{h} \alpha_{f}+\left(k^{2} l_{2}+2 n_{1}\right) \beta_{h} \beta_{f}-4\left[k^{2}\left(l_{2}+3 l_{3}+9 l_{4}+3 l_{5}\right)+3 n_{1}+9 n_{2}\right] \mathcal{R}_{h} \mathcal{R}_{f} \\
& -4\left[k^{2}\left(l_{2}+l_{3}+l_{4}+l_{5}\right)+n_{1}+n_{2}\right] \mathcal{E}_{h} \mathcal{E}_{f}-4\left[k^{2}\left(3 l_{4}+l_{5}\right)+3 n_{2}\right) \alpha_{h} \mathcal{R}_{f}-4\left(k^{2}\left(l_{3}+3 l_{4}\right)+3 n_{2}\right] \alpha_{f} \mathcal{R}_{h} \\
& +4\left[k^{2}\left(l_{4}+l_{5}\right)+n_{2}\right] \alpha_{h} \mathcal{E}_{f}+4\left[k^{2}\left(l_{3}+l_{4}\right)+n_{2}\right] \alpha_{f} \mathcal{E}_{h}+4\left[k^{2}\left(l_{2}+l_{3}+3 l_{4}+3 l_{5}\right)+n_{1}+3 n_{2}\right] \mathcal{R}_{h} \mathcal{E}_{f} \\
& +4\left[k^{2}\left(l_{2}+3 l_{3}+3 l_{4}+l_{5}\right)+n_{1}+3 n_{2}\right] \mathcal{R}_{f} \mathcal{E}_{h} .
\end{aligned}
$$

\section{APPENDIX C: TWO PRIMARY CASE IN VECTOR SECTOR}

In this appendix, we investigate the Hamiltonian analysis in the case of two primary constraints in the vector sector, where

$$
\kappa_{f 2}=-2 \kappa_{f 1}+\frac{l_{2}^{2}}{4\left(2 \kappa_{h 1}+\kappa_{h 2}\right)}
$$

is satisfied. In this case, we have the following two primary constraints, which is defined by

$$
\mathcal{C}_{B_{i}^{f}}^{(1)} \equiv \pi_{B_{i}^{f}}-\frac{l_{2}}{2\left(2 \kappa_{h 1}+\kappa_{h 2}\right)} \pi_{B_{i}^{h}} \approx 0
$$

Then we define the total Hamiltonian

$$
\mathcal{H}_{T}^{V}=\mathcal{H}^{V}+\lambda_{B_{i}^{f}} \mathcal{C}_{B_{i}^{f}}^{(1)}
$$

The consistency of the primary constraints gives the secondary constraints

$$
\begin{aligned}
\mathcal{C}_{B_{i}^{f}}^{(2)} \equiv\left\{\mathcal{C}_{B_{i}^{f}}^{(2)}, \mathcal{H}_{T}^{V}\right\}= & \left(4 \mu_{f 1}-\frac{l_{2} n_{1}}{2 \kappa_{h 1}+\kappa_{h 2}}\right) B_{i}^{f} \\
& +2\left(n_{1}-\frac{l_{2} \mu_{h 1}}{2 \kappa_{h 1}+\kappa_{h 2}}\right) B_{i}^{h} \\
& -k \pi_{F_{i}^{f}}+\frac{k l_{2}}{2\left(2 \kappa_{h 1}+\kappa_{h 2}\right)} \pi_{F_{i}^{h}},
\end{aligned}
$$

and the time evolution of the secondary constraints gives

$$
\mathcal{C}_{B_{i}^{f}}^{(3)} \equiv\left\{\mathcal{C}_{B_{i}^{f}}^{(2)}, \mathcal{H}_{T}^{V}\right\}=\left\{\mathcal{C}_{B_{i}^{f}}^{(2)}, \mathcal{H}^{V}\right\}+\lambda_{B_{i}^{f}}\left\{\mathcal{C}_{B_{i}^{f}}^{(2)}, \mathcal{C}_{B_{i}^{f}}^{(1)}\right\} \approx 0
$$

where

$$
\left\{\mathcal{C}_{B_{i}^{f}}^{(2)}, \mathcal{C}_{B_{i}^{f}}^{(1)}\right\}=4 \mu_{f 1}+\frac{l_{2}\left(l_{2} \mu_{h 1}-2 n_{1}\left(2 \kappa_{h 1}+\kappa_{h 2}\right)\right)}{\left(2 \kappa_{h 1}+\kappa_{h 2}\right)^{2}}
$$

Therefore, when $\left\{\mathcal{C}_{B_{i}^{f}}^{(2)}, \mathcal{C}_{B_{i}^{f}}^{(1)}\right\} \neq 0$, the Lagrange multipliers $\lambda_{B_{i}^{f}}$ are determined by the above equation, and the primary and secondary constraints are second class. Therefore, the number of the physical DOFs in the vector sector is $(8 \times 2-4) / 2=6$.

In order to further reduce the variable in the phase space, we need to impose an extra condition. The only option here is $\left\{\mathcal{C}_{B_{i}^{f}}^{(2)}, \mathcal{C}_{B_{i}^{f}}^{(1)}\right\}=0$, i.e.,

$$
\mu_{f 1}=-\frac{l_{2}\left(l_{2} \mu_{h 1}-2 n_{1}\left(2 \kappa_{h 1}+\kappa_{h 2}\right)\right)}{4\left(2 \kappa_{h 1}+\kappa_{h 2}\right)^{2}} .
$$

Then, $\mathcal{C}_{B_{i}^{f}}^{(3)}$ serves as the tertiary constraints,

$$
\mathcal{C}_{B_{i}^{f}}^{(3)}=\frac{\left[n_{1}\left(2 \kappa_{h 1}+\kappa_{h 2}\right)-l_{2} \mu_{h 1}\right]\left[k l_{2} F_{i}^{f}+2 k\left(2 \kappa_{h 1}+\kappa_{h 2}\right) F_{i}^{h}-\pi_{B_{i}^{h}}\right]}{\left(2 \kappa_{h 1}+\kappa_{h 2}\right)^{2}} \approx 0
$$

and, since $\left\{\mathcal{C}_{B_{i}^{f}}^{(3)}, \mathcal{C}_{B_{i}^{f}}^{(1)}\right\}=0$, subsequently we have quaternary constraints,

$$
\mathcal{C}_{B_{i}^{f}}^{(4)} \equiv\left\{\mathcal{C}_{B_{i}^{f}}^{(3)}, \mathcal{H}_{T}^{V}\right\}=-\frac{\left[n_{1}\left(2 \kappa_{h 1}+\kappa_{h 2}\right)-l_{2} \mu_{h 1}\right]\left[\left(k^{2} l_{2}+2 n_{1}\right) B_{i}^{f}+2\left(k^{2}\left(2 \kappa_{h 1}+\kappa_{h 2}\right)+2 \mu_{h 1}\right) B_{i}^{h}-k \pi_{F_{i}^{h}}\right]}{\left(2 \kappa_{h 1}+\kappa_{h 2}\right)^{2}} \approx 0 .
$$


Now the time evolution of the quaternary constraints gives

$\dot{\mathcal{C}}_{B_{i}^{f}}^{(4)}=\left\{\mathcal{C}_{B_{i}^{f}}^{(4)}, \mathcal{H}_{T}^{V}\right\}=\left\{\mathcal{C}_{B_{i}^{f}}^{(4)}, \mathcal{H}^{V}\right\}+\lambda_{B_{f, i}}\left\{\mathcal{C}_{B_{f, i}}^{(4)}, \mathcal{C}_{B_{i}^{f}}^{(1)}\right\} \approx 0$,

where

$$
\left\{\mathcal{C}_{B_{i}^{f}}^{(4)}, \mathcal{C}_{B_{i}^{f}}^{(1)}\right\}=-\frac{2\left(n_{1}\left(2 \kappa_{h 1}+\kappa_{h 2}\right)-l_{2} \mu_{h 1}\right)^{2}}{\left(2 \kappa_{h 1}+\kappa_{h 2}\right)^{3}} .
$$

Therefore, as long as $\left\{\mathcal{C}_{B_{i}^{f}}^{(4)}, \mathcal{C}_{B_{i}^{f}}^{(1)}\right\}$ is nonvanishing, the Lagrange multiplier is determined by the above equations, and the number of the physical DOFs is $(8 \times 2-8) / 2=4$.

When $n_{1}\left(2 \kappa_{h 1}+\kappa_{h 2}\right)-l_{2} \mu_{h 1}=0$, the above tertiary constraint trivially vanishes and its time evolution does not generate the independent constraint. In this case, we only have the primary and secondary constraints, but now they are first class since all the primary and secondary constraints commute each other. Thus, the number of the physical DOFs is $(8 \times 2-4 \times 2) / 2=4$. Therefore, the case with two primary constraints in the vector sector cannot have 2 physical DOFs.

\section{APPENDIX D: TWO PRIMARY CASE: $\operatorname{det} \mathcal{K}^{S} \neq 0$ IN SCALAR SECTOR}

Let us consider the case with two primary constraints, namely the degenerate condition for the scalar components is not imposed, and the parameters only satisfy the vector conditions (44). We define the following two primary constraints for convenience:

$$
\mathcal{C}_{\beta_{h}}^{(1)} \equiv \pi_{\beta_{h}} \approx 0, \quad \mathcal{C}_{\beta_{f}}^{(2)} \equiv \pi_{\beta_{f}}-\frac{n_{1}}{2 \mu_{h 1}} \pi_{\beta_{h}} \approx 0 .
$$

The total Hamiltonian is defined as

$$
\mathcal{H}_{T}^{S}=\mathcal{H}+\lambda_{\beta_{h}} \mathcal{C}_{\beta_{h}}^{(1)}+\lambda_{\beta_{f}} \mathcal{C}_{\beta_{f}}^{(1)}
$$

The evolution of the two primary constraints yields two secondary constraints:

$$
\begin{aligned}
\mathcal{C}_{\beta_{h}}^{(2)} & =\left\{\mathcal{C}_{\beta_{h}}^{(1)}, \mathcal{H}_{T}^{S}\right\} \\
& =-k\left(\pi_{\alpha_{h}}+\pi_{\mathcal{E}_{h}}\right)+4 \mu_{h 1} \beta_{h}+2 n_{1} \beta_{f} \approx 0, \\
\mathcal{C}_{\beta_{f}}^{(2)} & =\left\{\tilde{\mathcal{C}}_{\beta_{f}}^{(1)}, \mathcal{H}_{T}^{S}\right\} \\
& =-k\left(\pi_{\alpha_{f}}+\pi_{\mathcal{E}_{f}}\right)+\frac{k n_{1}}{2 \mu_{h 1}}\left(\pi_{\alpha_{h}}+\pi_{\mathcal{E}_{h}}\right) \approx 0 .
\end{aligned}
$$

Since $\left\{\mathcal{C}_{\beta_{h}}^{(2)}, \mathcal{C}_{\beta_{h}}^{(1)}\right\}=4 \mu_{h 1} \neq 0$, the Lagrange multiplier $\lambda_{\beta_{h}}$ is determined by imposing $\dot{\mathcal{C}}_{\beta_{h}}^{(2)} \approx 0$, namely $\lambda_{\beta_{h}} \approx$ $-\left\{\mathcal{C}_{\beta_{h}}^{(2)}, \mathcal{H}\right\} /\left\{\mathcal{C}_{\beta_{h}}^{(2)}, \mathcal{C}_{\beta_{h}}^{(1)}\right\}$. The evolution of the remaining secondary constraint yields the tertiary constraint:

$\mathcal{C}_{\beta_{f}}^{(3)} \equiv\left\{\mathcal{C}_{\beta_{f}}^{(2)}, \mathcal{H}_{T}^{S}\right\}=2 k^{3}\left[-2 l_{5} \alpha_{h}+\frac{l_{3} n_{1}}{\mu_{h 1}} \alpha_{f}-2\left(3 l_{5}-\frac{2 n_{1} \kappa_{h 1}}{\mu_{h 1}}\right) \mathcal{R}_{h}-\left(8 \kappa_{f 1}-\frac{3 l_{3} n_{1}}{\mu_{h 1}}\right) \mathcal{R}_{f}+2 l_{5} \mathcal{E}_{h}-\frac{l_{3} n_{1}}{\mu_{h 1}} \mathcal{E}_{f}\right] \approx 0$.

Here, the tertiary constraint cannot be trivially zero since $\kappa_{f 1} \neq 0$. One can also check that $\dot{\mathcal{C}}_{\beta_{f}}^{(3)}=k^{2} \mathcal{C}_{\beta_{f}}^{(2)} \approx 0$, implying no more constraint is generated. The constraints $\mathcal{C}_{\beta_{f}}^{(1,2,3)}$ commute with all other constraints, and therefore, we have three first-class constraints $\mathcal{C}_{\beta_{f}}^{(1,2,3)}$ and two second-class constraints $\mathcal{C}_{\beta_{h}}^{(1,2)}$. Hence, the number of the physical DOFs is $(8 \times 2-2-3 \times 2) / 2=4$. Since there is no further option to eliminate DOFs, one cannot obtain 1 DOF theory in this case.

\section{APPENDIX E: EXPLICIT EXPRESSION OF CONSTRAINTS}

In this appendix, we give an explicit expression of the constraints in the case of Class I. Equation (69) is given by

$$
\mathcal{C}_{\alpha_{f}}^{(3)} \equiv\left\{\mathcal{C}_{\alpha_{f}}^{(2)}, \mathcal{H}_{T}^{S}\right\}=-\frac{8 k l_{3} \mu_{h 1}}{\kappa_{h 1}} \beta_{h}+c_{3}^{\alpha_{h}} \pi_{\alpha_{h}}+c_{3}^{\mathcal{R}_{h}} \pi_{\mathcal{R}_{h}}+c_{3}^{\mathcal{R}_{f}} \pi_{\mathcal{R}_{f}}+\frac{k^{2} l_{3}}{\kappa_{h 1}} \pi_{\mathcal{E}_{h}}+k^{2} \pi_{\mathcal{E}_{f}} \approx 0
$$

where

$$
c_{3}^{\alpha_{h}}=\frac{1}{4 \kappa_{h 1}}\left[l_{3}\left(k^{2}+\frac{6 \mu_{h 1}}{\kappa_{h 1}}\right)-\left(16 \kappa_{h 1} \kappa_{f 1}+9 l_{3}^{2}\right) y\right],
$$

$$
\begin{gathered}
c_{3}^{\mathcal{R}_{h}}=\frac{l_{3}}{4 \kappa_{h 1}}\left(k^{2}-\frac{2 \mu_{h 1}}{\kappa_{h 1}}+3 l_{3} y\right), \\
c_{3}^{\mathcal{R}_{f}}=-l_{3} y,
\end{gathered}
$$


and

$$
y=\frac{2 n_{2} \kappa_{h 1}+3 l_{3} \mu_{h 1}}{2 \kappa_{h 1}\left(8 \kappa_{f 1}\left(\kappa_{h 1}+\kappa_{h 4}\right)+3 l_{3}^{2}\right)} .
$$

$$
\begin{aligned}
\tilde{c}_{4}^{\alpha_{f}}= & \frac{1}{\kappa_{h 1}^{2}}\left[-2 l_{3}\left(4 \kappa_{h 1} \kappa_{f 1}+3 l_{3}^{2}\right)\left(2 \kappa_{h 1} k^{2}+3 \mu_{h 1}\right) y\right. \\
& \left.+8 \kappa_{f 1}\left(2 \kappa_{h 1} n_{2}+3 l_{3} \mu_{h 1}\right) y-2 k^{2} l_{3}^{2}\left(2 k^{2} \kappa_{h 1}-\mu_{h 1}\right)\right],
\end{aligned}
$$

After rescaling, Eq. (70) is given by

$$
\begin{aligned}
\tilde{\mathcal{C}}_{\alpha_{f}}^{(3)} & \equiv\left\{\mathcal{C}_{\alpha_{f}}^{(2)}, \mathcal{H}_{T}^{S}\right\} \\
& =\tilde{c}_{3}^{\alpha_{h}} \pi_{\alpha_{h}}+\tilde{c}_{3}^{\mathcal{R}_{h}} \pi_{\mathcal{R}_{h}}+c_{3}^{\mathcal{R}_{f}} \pi_{\mathcal{R}_{f}}-\frac{k^{2} l_{3}}{\kappa_{h 1}} \pi_{\mathcal{E}_{h}} \approx 0,
\end{aligned}
$$

where

$$
\begin{aligned}
& \tilde{c}_{3}^{\alpha_{h}}= c_{3}^{\alpha_{h}}-\frac{5 k^{2} l_{3}}{4 \kappa_{h 1}} \\
&= \frac{1}{4 \kappa_{h 1}}\left[l_{3}\left(-4 k^{2}+\frac{6 \mu_{h 1}}{\kappa_{h 1}}\right)-\left(16 \kappa_{h 1} \kappa_{f 1}+9 l_{3}^{2}\right) y\right], \\
& \tilde{c}_{3}^{\mathcal{R}_{h}}=c_{3}^{\mathcal{R}_{h}}-\frac{k^{2} l_{3}}{4 \kappa_{h 1}}=\frac{l_{3}}{4 \kappa_{h 1}}\left(-\frac{2 \mu_{h 1}}{\kappa_{h 1}}+3 l_{3} y\right) .
\end{aligned}
$$

The time evolution of $\tilde{\mathcal{C}}_{\alpha_{f}}^{(3)}$ yields the constraint

$$
\begin{aligned}
\dot{\tilde{\mathcal{C}}}_{\alpha_{f}}^{(3)} \equiv \tilde{\mathcal{C}}_{\alpha_{f}}^{(4)}= & \tilde{c}_{4}^{\alpha_{h}} \alpha_{h}+\tilde{c}_{4}^{\alpha_{f}} \alpha_{f}+\tilde{c}_{4}^{\mathcal{E}_{h}} \mathcal{E}_{h}+\tilde{c}_{4} \mathcal{E}_{f} \mathcal{E}_{f}+\tilde{c}_{4}^{\mathcal{R}_{h}} \mathcal{R}_{h} \\
& +\tilde{c}_{4}^{\mathcal{R}_{f}} \mathcal{R}_{f} \approx 0
\end{aligned}
$$

$$
\begin{aligned}
\tilde{c}_{4}^{\mathcal{E}_{h}=} & -8\left(4 \kappa_{f 1}\left(\kappa_{h 1}+\kappa_{h 4}\right)+l_{3}^{2}\right) k^{2} y \\
& +2\left(\frac{3 l_{3}}{\kappa_{h 1}}\left(-2 \kappa_{h 1} n_{2}+l_{3} \mu_{h 1}\right)-16 \kappa_{f 1} \mu_{h 2}\right) y \\
& +\frac{4 l_{3}\left(2 k^{2} \kappa_{h 1}-\mu_{h 1}\right) \mu_{h 1}}{\kappa_{h 1}^{2}}, \\
\tilde{c}_{4} \mathcal{E}_{f}= & \frac{4 l_{3}\left(4 \kappa_{h 1} \kappa_{f 1}+3 l_{3}^{2}\right)}{\kappa_{h 1}} k^{2} y+2\left(-8 \kappa_{f 1} n_{2}+\frac{9 l_{3}^{3} \mu_{h 1}}{\kappa_{h 1}^{2}}\right) y \\
& +\frac{2 k^{2} l_{3}^{2}\left(2 k^{2} \kappa_{h 1}-\mu_{h 1}\right)}{\kappa_{h 1}^{2}}, \\
\tilde{c}_{4}^{\mathcal{R}_{h}}= & 8\left(4 \kappa_{f 1}\left(\kappa_{h 1}+3 \kappa h 4\right)-4 l_{3}^{2}\right) k^{2} y \\
& +2\left(\frac{9 l_{3}}{\kappa_{h 1}}\left(2 \kappa_{h 1} n_{2}-l_{3} \mu_{h 1}\right)+48 \kappa_{f 1} \mu_{h 2}\right) y \\
& -\frac{4 l_{3}\left(4 k^{4} \kappa_{h 1}^{2}-2 k^{2} \kappa_{h 1} \mu_{h 1}-3 \mu_{h 1}^{2}\right)}{\kappa_{h 1}^{2}},
\end{aligned}
$$

where

$$
\begin{aligned}
\tilde{c}_{4}^{\alpha_{h}}= & 8\left(4 \kappa_{f 1}\left(\kappa_{h 1}+\kappa_{h 4}\right)+l_{3}^{2}\right) k^{2} y \\
& +2\left(\frac{3 l_{3}}{\kappa_{h 1}}\left(2 \kappa_{h 1} n_{2}+3 l_{3} \mu_{h 1}\right)+16 \kappa_{f 1}\left(\mu_{h 1}+\mu_{h 2}\right)\right) y \\
& -\frac{12 l_{3} \mu_{h 1}^{2}}{\kappa_{h 1}^{2}},
\end{aligned}
$$

$$
\begin{aligned}
\tilde{c}_{4}^{\mathcal{R}_{f}}= & -\frac{4 l_{3}\left(8 \kappa_{h 1} \kappa_{f 1}+9 l_{3}^{2}\right)}{\kappa_{h 1}} k^{2} y \\
& +6\left(8 \kappa_{f 2} n_{2}-\frac{9 l_{3}^{2} \mu_{h 1}}{\kappa_{h 1}^{2}}\right) y-\frac{6 k^{2} l_{3}^{2}\left(2 k^{2} \kappa_{h 1}-\mu_{h 1}\right)}{\kappa_{h 1}^{2}} .
\end{aligned}
$$

Using other constraints, we then rewrite $\tilde{\mathcal{C}}_{\alpha_{f}}^{(4)}$ as

$$
\begin{aligned}
\tilde{\mathcal{C}}_{\alpha_{f}}^{(4)}= & \frac{2 n_{2} \kappa_{h 1}+3 l_{3} \mu_{h 1}}{\kappa_{h 1}\left(8 \kappa_{f 1}\left(\kappa_{h 1}+\kappa_{h 4}\right)+3 l_{3}^{2}\right)}\left[\frac{l_{3}}{2} \mathcal{C}_{\alpha_{f}}^{(2)}-\frac{6 l_{3}^{3} \mu_{h 1}^{2}+\kappa_{f 1}^{2} \kappa_{f 1}\left(k^{2}\left(2 \kappa_{h 1} n_{2}+3 l_{3} \mu_{h 1}\right)-4 n_{2} \mu_{h 1}\right)}{3 k l_{3}^{2} \mu_{h 1}^{2}} \mathcal{C}_{\beta_{f}}^{(3)}\right. \\
& \left.-16 \kappa_{f 1}\left(\mu_{h 2}+\frac{\kappa_{h 1}^{2} n_{2}^{2}}{3 l_{3}^{2} \mu_{h 1}}+\frac{1}{4} \mu_{h 1}\right)\left(\mathcal{E}_{h}-\alpha_{h}-3 \mathcal{R}_{h}\right)\right] \\
& +\frac{1}{\kappa_{h 1}^{2}\left(2 k^{2} \kappa_{h 1}+3 \mu_{h 1}\right)}\left(1-\frac{\kappa_{h 1} \kappa_{f 1}\left(2 n_{2} \kappa_{h 1}+3 l_{3} \mu_{h 1}\right)^{2}}{3 l_{3}^{2} \mu_{h 1}^{2}\left(8 \kappa_{f 1}\left(\kappa_{h 1}+\kappa_{h 4}\right)+3 l_{3}^{2}\right)}\right)\left[k \kappa_{h 1}^{2}\left(2 k^{2} \kappa_{h 1}-\mu_{h 1}\right) \mathcal{C}_{\beta_{f}}^{(3)}\right. \\
& \left.+4 \mu_{h 1}\left(4 k^{2} \kappa_{h 1}^{2} n_{2}+3 l_{3} \mu_{h 1}^{2}\right)\left(\mathcal{E}_{h}-\alpha_{h}-3 \mathcal{R}_{h}\right)-48 l_{3} \mu_{h 1}^{2}\left(2 k^{2} \kappa_{h 1} \mathcal{R}_{h}-\mu_{h 1} \alpha_{h}\right)\right] .
\end{aligned}
$$

When we further impose the two additional conditions (73) and (86), the right-hand side of the above equation reduces to the linear combination of the constraints, namely the time evolution of $\tilde{\mathcal{C}}_{\alpha_{f}}^{(3)}$ becomes trivial. 
[1] C. de Rham and G. Gabadadze, Phys. Rev. D 82, 044020 (2010).

[2] M. Fierz and W. Pauli, Proc. R. Soc. A 173, 211 (1939).

[3] V. I. Zakharov, Pis'ma Zh. Eksp. Teor. Fiz. 12, 447 (1970) [JETP Lett. 12, 312 (1970)], https://www.osti.gov/biblio/ 4050748-linearized-gravitation-theory-graviton-mass.

[4] H. van Dam and M. J. G. Veltman, Nucl. Phys. B22, 397 (1970).

[5] A. I. Vainshtein, Phys. Lett. 39B, 393 (1972).

[6] D. G. Boulware and S. Deser, Phys. Rev. D 6, 3368 (1972).

[7] M. V. Ostrogradsky, Mem. Acad. St. Petersbourg VI 4, 385 (1850), https://inspirehep.net/literature/1468685.

[8] C. de Rham, G. Gabadadze, and A. J. Tolley, Phys. Rev. Lett. 106, 231101 (2011).

[9] A. E. Gumrukcuoglu, C. Lin, and S. Mukohyama, J. Cosmol. Astropart. Phys. 11 (2011) 030.

[10] A. De Felice, A. E. Gumrukcuoglu, and S. Mukohyama, Phys. Rev. Lett. 109, 171101 (2012).

[11] A. E. Gumrukcuoglu, C. Lin, and S. Mukohyama, J. Cosmol. Astropart. Phys. 03 (2012) 006.

[12] K. Hinterbichler, J. High Energy Phys. 10 (2013) 102.

[13] R. Kimura and D. Yamauchi, Phys. Rev. D 88, 084025 (2013).
[14] C. de Rham, A. Matas, and A. J. Tolley, Classical Quantum Gravity 31, 165004 (2014).

[15] A. E. Gümrükçüoglu and K. Koyama, Phys. Rev. D 99, 084004 (2019).

[16] C. De Rham, L. Keltner, and A. J. Tolley, Phys. Rev. D 90, 024050 (2014).

[17] M. Kenna-Allison, A. E. Gumrukcuoglu, and K. Koyama, Phys. Rev. D 101, 084014 (2020).

[18] A. E. Gumrukcuoglu, R. Kimura, and K. Koyama, Phys. Rev. D 101, 124021 (2020).

[19] S. F. Hassan and R. A. Rosen, J. High Energy Phys. 02 (2012) 126.

[20] S. Hassan and R. A. Rosen, J. High Energy Phys. 04 (2012) 123.

[21] A. Naruko, R. Kimura, and D. Yamauchi, Phys. Rev. D 99, 084018 (2019).

[22] R. Sugano and H. Kamo, Prog. Theor. Phys. 67, 1966 (1982).

[23] R. Sugano, Y. Saito, and T. Kimura, Prog. Theor. Phys. 76, 283 (1986).

[24] R. Sugano and T. Kimura, Phys. Rev. D 41, 1247 (1990). 